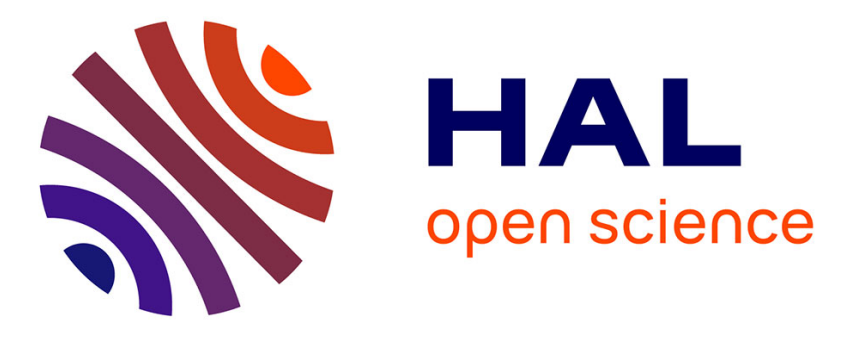

\title{
Tracking vehicle trajectories and fuel rates in phantom traffic jams: Methodology and data
}

Fangyu Wu, Raphael E. Stern, Shumo Cui, Maria Laura Delle Monache, Rahul Bhadani, Matt Bunting, Miles Churchill, Nathaniel Hamilton, R'Mani Haulcy, Benedetto Piccoli, et al.

\section{To cite this version:}

Fangyu Wu, Raphael E. Stern, Shumo Cui, Maria Laura Delle Monache, Rahul Bhadani, et al.. Tracking vehicle trajectories and fuel rates in phantom traffic jams: Methodology and data. Transportation research. Part C, Emerging technologies, 2019, 99, pp.82-109. 10.1016/j.trc.2018.12.012 . hal-01614665

\section{HAL Id: hal-01614665 \\ https://hal.inria.fr/hal-01614665}

Submitted on 11 Oct 2017

HAL is a multi-disciplinary open access archive for the deposit and dissemination of scientific research documents, whether they are published or not. The documents may come from teaching and research institutions in France or abroad, or from public or private research centers.
L'archive ouverte pluridisciplinaire HAL, est destinée au dépôt et à la diffusion de documents scientifiques de niveau recherche, publiés ou non, émanant des établissements d'enseignement et de recherche français ou étrangers, des laboratoires publics ou privés. 


\title{
Tracking vehicle trajectories and fuel rates in oscillatory traffic
}

\author{
Fangyu $\mathrm{Wu}^{\mathrm{a}}$, Raphael E. Stern ${ }^{\mathrm{a}}$, Shumo Cui ${ }^{\mathrm{b}}$, Maria Laura Delle Monache ${ }^{\mathrm{c}}$, Rahul Bhadani ${ }^{\mathrm{d}}$, Matt \\ Bunting $^{\mathrm{d}}$, Miles Churchill ${ }^{\mathrm{a}}$, Nathaniel Hamilton ${ }^{\mathrm{e}}$, R'mani Haulcy ${ }^{\mathrm{f}}$, Benedetto Piccoli ${ }^{\mathrm{g}}$, Benjamin Seibold ${ }^{\mathrm{b}}$, \\ Jonathan Sprinkle ${ }^{\mathrm{d}}$, Daniel B. Work ${ }^{\mathrm{a}, *}$ \\ ${ }^{a}$ Department of Civil Engineering, University of Illinois at Urbana-Champaign, 205 N. Mathews Ave, Urbana, IL 61801, \\ USA. \\ ${ }^{b}$ Department of Mathematics, Temple University, 1805 North Broad Street, Philadelphia, PA 19122, USA. \\ ${ }^{c}$ Inria, University Grenoble Alpes, CNRS, GIPSA-lab, F-38000 Grenoble, France. \\ ${ }^{d}$ Electrical and Computer Engineering, University of Arizona, Tucson, AZ 85721-0104, USA. \\ ${ }^{e}$ Lipscomb University, 1 University Park Drive, Nashville, TN 37204, USA. \\ ${ }^{f}$ Yale University, New Haven, CT 06520, USA. \\ ${ }^{g}$ Department of Mathematical Sciences, Rutgers University-Camden, 311 N. 5th St, Camden, NJ 08102, USA.
}

\begin{abstract}
High-fidelity vehicle trajectory data is becoming increasingly important in traffic modeling, especially to capture dynamic features such as stop-and-go waves. This article presents data collected in a series of eight experiments on a circular track with human drivers. The data contains smooth flowing and stop-and-go traffic conditions. The vehicle trajectories presented in this article are collected using a panoramic 360degree camera, and fuel rate data is recorded via an on-board diagnostics scanner installed in each vehicle. The video data from the 360-degree camera is processed with an offline unsupervised algorithm to extract vehicle trajectories from experimental data. The trajectories are highly accurate, with a mean positional bias of less than $0.01 \mathrm{~m}$ and a standard deviation of $0.11 \mathrm{~m}$. The velocities are also validated to be highly accurate with a bias of $0.02 \mathrm{~m} / \mathrm{s}$ and standard deviation of $0.09 \mathrm{~m} / \mathrm{s}$. The source code and data used in this article are published with this work.
\end{abstract}

Keywords: traffic waves, fuel rate, vehicle trajectories, computer vision, open datasets

\section{Introduction}

\subsection{Motivation: The need for empirical traffic data}

For many decades, the collection and interpretation of empirical traffic data has shaped our understanding of vehicular interactions and traffic flow. The first major data collection effort began with the pioneering experiments conducted by Greenshields [1] in the 1930's, which provided an empirical model between the density and velocity of traffic and consequently the construction of the fundamental diagram. In the late 1960's and 1970's automated systems were deployed to collect aggregated data for the purpose of monitoring and controlling traffic in the Lincoln Tunnel [2. Other systems were deployed to estimate traffic conditions on the Long Island Expressway in New York [3, and to estimate traffic flow model parameters [4.

Today, the shape of fundamental diagrams and more broadly the aggregated or macroscopic dynamics of traffic are greatly aided by the abundance of traffic data measured from fixed sensors such as inductive loop detectors, radars, and video cameras. One prevalent traffic data source in the US is the Performance Measurement System (PeMS) database [5]. This database collects data from nearly 40,000 inductive loop

\footnotetext{
* Corresponding author

Email address: dbwork@illinois.edu (Daniel B. Work)
} 
detectors and toll tag readers in the state of California. Similarly, the Grenoble Traffic lab has instrumented a road in Grenoble, France with magnetic sensors embedded in the roadway to collect vehicle counts [6], while the Minnesota Traffic Observatory uses video detectors on the I-35W/I-94 freeway to collect vehicle counts and trajectories [7. The Berkeley Highway Lab collected traffic counts using loop detectors on I- 80 in Emeryville, CA 8 . Such systems typically provide vehicle counts or flows, time averaged velocities, and time averaged occupancies that enable the estimation of the traffic density.

One important limitation of such datasets is that they are initially introduced to collect aggregated data relevant for calibrating and validating macroscopic descriptions of the traffic flow [9, 10, 11, 12, Microscopic descriptions of traffic [13, 14, 15, 16, 17] can be challenging to validate in detail without data at the level of the individual vehicle.

In parallel with the aggregated data collection efforts, early efforts aimed at measuring individual vehicle trajectories include the work of Treiterer [18 in the 1970s. The experiments involved flying a helicopter over freeways in Ohio to photograph the traffic, which is later used to reconstruct the vehicle trajectories.

The experiments by Treiterer served as the first in a series of larger efforts to collect vehicle trajectories. Coifman [19] collected trajectories using a video camera on a $120 \mathrm{~m}$ segment of I-680 in California and observed the development of shockwaves, while other US Federal Highway Administration efforts used video footage from an aircraft to collect vehicle trajectories at six types of freeway bottleneck sections [20]. Researchers at Delft University have used cameras mounted on a helicopter to collect vehicle trajectory data on a $520 \mathrm{~m}$ freeway segment [21] in Utrecht.

Amongst the most widely used trajectory datasets are the NGSIM datasets, which are collected "in support of traffic simulation with a primary focus on microscopic modeling," 22. The datasets contain vehicular trajectories recorded at different times on three different road segments. The first of these datasets was collected using video cameras on a $900 \mathrm{~m}$ segment of freeway I-80 in California over 30 minutes in December of 2003. Additionally, in April of 2005, three further 15 minute data collections were performed on a $500 \mathrm{~m}$ segment of the same highway. Also in 2005, a $640 \mathrm{~m}$ segment of US-101 in Los Angeles was instrumented, and three consecutive 15 minute datasets were recorded. Several recent works have used the NGSIM data to calibrate traffic models [23, 24, 25, 26, 27, 28. However, as pointed out in some recent works, there are limitations to this dataset since the acceleration and velocity measurements are prone to large errors [29, 30, 31, due to the finite difference calculation on the successive vehicle positions. Other experimental efforts have been able to collect vehicle trajectories from individual vehicles in urban traffic [32, 33. and instrumented platoons of vehicles 34.

At the individual vehicle level, driver behavior has been analyzed using data collected through the naturalistic driving study (NDS) [35. The data contain video footage and vehicle performance data from instrumented vehicles, and includes both a driver-facing and a forward-facing camera. The dataset contains over 42,300 hours and 2,000,000 miles of driving. Additionally, these data include 82 collisions and 761 near misses, which have been used to analyze driving risks [36, 37, 38. A related naturalistic driving dataset has also been created by the University of Michigan with data for nearly one million vehicle miles traveled [39]. While the datasets provide extensive information about the instrumented vehicles, a limitation is that they do not include information regarding all vehicles in the traffic steam.

The Mobile Century project at the University of California - Berkeley collected smartphone-based GPS position data from probe vehicles on I-880 near Oakland, California [40, but only for a subset of the total vehicles in the flow.

Other frequently used datasets for microscopic traffic modelling includes those collected by Sugiyama, et al. 41] and Tadaki, et al. [42. These datasets are fundamentally different from the previously mentioned traffic datasets since it includes data from closed-road traffic experiments as opposed to data collected on public roadways. In order to experimentally demonstrate the development of traffic instabilities such as stop-and-go waves even without lane changes or bottlenecks, Sugiyama, et al. [41] designed and executed a set of experiments in 2007, and later in 2013 [42. These experiments involved between 10 and 40 vehicles on a circular track. All vehicles begin with a uniform velocity and spacing, but the traffic quickly develops instabilities such as stop-and-go waves. This dataset, and other similar datasets 42, 43, 44 are also used for calibrating microscopic traffic flow models [17, 44, 45, 46]. For example, Jiang, et al. [44 collected vehicle trajectories from a platoon of 25 vehicles using GPS sensors and used the data to calibrate the parameters 
of the Intelligent Driver Model (IDM) 44. However, due to the resolution of the GPS receiver, the vehicle position accuracy is limited to $1 \mathrm{~m}$.

The experiments conducted by Sugiyama, et al. 41, 42 provide valuable vehicle trajectories, but does not contain any information on engine performance such as fuel rates. Fuel rates is important when studying the effects of speed oscillations on environmental factors such as emissions. Furthermore, additional open trajectory datasets under oscillatory traffic may prove useful for calibrating traffic models and designing autonomous vehicle (AV) controllers [47, 48, 49, 50, 51, 52, 53, 54, 55, 56, 57, 58, 59, 60, for driving in the presence of stop and go waves, or controlling the vehicle to eliminate them.

\subsection{Contributions and outline}

The first and main contribution of this article is the collection of eight experimental datasets that that contain accurate and high resolution vehicle trajectories and instantaneous fuel rates in the ring setting of Sugiyama et al. 41, 42, which is known to create stop-and-go waves. The trajectories are extracted from a 360-degree panoramic camera placed at the center of a circular track. Engine performance data such as engine speed and fuel rate are collected from an OBD-II scanner installed in each vehicle. The full dataset is published with this article 61.

A second contribution of the work is the development of an unsupervised data processing technique to extract trajectories from video data. In the pioneering experiments in Sugiyama et al. 41, the position of each vehicle is determined accurate to within $\pm 0.5 \mathrm{~m}$ at $3 \mathrm{~Hz}$, yielding a velocity error of $\pm 3 \mathrm{~m} / \mathrm{s}$ (roughly $30 \%$ of the target vehicle velocity of $8.33 \mathrm{~m} / \mathrm{s}$ ) 62. In a follow up experiment [42, a laser scanner is used to locate the vehicles at a $0.16 \mathrm{~m}$ spatial resolution and $0.2 \mathrm{~s}$ in time, improving the accuracy compared to the earlier tests. In our work we provide a low cost method to extract trajectories relying only on a 360-degree camera (less than \$400) and an unsupervised image processing algorithm. The resulting trajectories are validated and shown to have a positive bias of less than $0.01 \mathrm{~m}$ with a standard deviation of $0.11 \mathrm{~m}$; the velocity has a mean error of $0.02 \mathrm{~m} / \mathrm{s}$ with a standard deviation of $0.09 \mathrm{~m} / \mathrm{s}$. This high accuracy renders the resulting data useful particularly for microsimulation validation on an individual vehicle trajectory level, involving sharp braking profiles. The corresponding source code used to extract vehicle trajectories is also provided with this work 63 .

Although the primary focus of the work is the construction of the dataset, we also provide observations of the significant events happened during experiments to aid in further investigation. We note the extent to which oscillatory traffic is realized depends, partially, on the driver behavior resulting from the instruction set used to brief the drivers.

The remainder of the article is organized as follows. In Section 2 the experimental setup is explained, and a list of experiments is presented. In Section 3 the video data processing pipeline to extract trajectories from 360-degree panoramic video footage is described. Experimental measurements are validated in Section 4 to ensure they are accurate, and the experimental data along with trends found in the data are presented in Section 5 . Finally, we conclude that this new dataset provides new opportunities in traffic modelling in Section 6

\section{Description of experiments}

In this section, a series of experiments with 19 to 22 vehicles are described with the objective to measure the fuel consumption rates in stop-and-go traffic. In the interest of repeatability, the experimental setup is briefly outlined in Section 2.1 and the experimental protocol is summarized in Section 2.2

A total of eight experiments are conducted in Tucson, Arizona in July 2016. The goals of these experiments are two-fold: $(i)$ to identify under what conditions (traffic density and driver instructions) oscillatory traffic will arise and (ii) to investigate the extent to which a single vehicle, driving differently from the remaining traffic, is able to change the traffic state.

Each experiment is labeled with a letter from $\mathrm{A}$ to $\mathrm{H}$, in the order they occurred. In each experiment drivers are given specific instructions on how to drive. Full experiment descriptions are provided in Section 2.2 


\subsection{Experimental setup}

To achieve the goals of the experiments as outlined above, we seek to re-create the results observed in the Sugiyama, et al. experiment [4] with the addition of instrumenting each individual vehicle with OBD-II scanners. Some experimental changes are made (track size, direction of driving) to account for larger and right-hand drive vehicles in the US compared to Japan. The track is available for a total of four hours for experimentation. Taking driver rest breaks and a driver briefing into account this allows for three hours of testing. The time to re-set the track after each experiment is approximately 15 minutes, and each experiment lasts between five and 10 minutes. Therefore, eight experiments are able to be completed.

The experiments are divided into two sets. In Experiments A-E, we use instruction set I for which each driver is instructed to "Safely follow the vehicle in front as if in rush hour traffic," and vary the density by changing the number of vehicles on the road. We visually observe that some vehicles drive very conservatively (e.g., by leaving excessive gaps) which dampens any waves that might arise. Consequently, we change the driving instructions of all drivers to instruction set II in Experiments F, G, and H, for which drivers are instructed to "Drive by the same instructions as before, but in addition place an emphasis on closing the gap the the vehicle in front, whenever safety permits." The complete verbal instructions given to the drivers at site are provided in the Appendix. Additionally, for Experiments G and $\mathrm{H}$ we instruct one driver (co-author M. Bunting, a member of experimental staff) to change his driving behavior during the experiment to observe the effect that a single vehicle has on the overall traffic flow.

The experiments are conducted on a circular track $260 \mathrm{~m}$ in circumference. The track length is selected to approximate the total unoccupied road space of the experiments conducted by Sugiyama et al. [41. The track is constructed on a large paved parking lot at the Tucson Dragway in Tucson, Arizona, and selected for its smooth, even surface, and abundance of open space. The experimental track with vehicles is shown in Figure 1a. The inside edge of the track is marked with short orange cones. Additional cones are used to mark the pre-measured location of the front tire of each car to ensure uniform spacing at the start of the experiment.

An air horn is used to signal the start of the experiment to the drivers. The first air horn is sounded to allow the drivers to shift into gear and prepare to drive. The next air horn is sounded to notify the drivers to start driving. Another air horn is sounded to notify the drivers that the experiment had ended, and they should safely come to a complete stop on the track.

The vehicles used for this experiment are procured from the University of Arizona's motor vehicle pool. The year, make, model, length, and nominal EPA-related fuel rate of each vehicle used in the experiment is presented in Table A.1. The vehicles used in each experiment is given in Table A.2.

\subsection{Experimental protocol}

The experimental protocol used is the same as that used in [64, with the critical difference that in [64] an autonomous vehicle is used to control the traffic stream, while the purpose of the experiments in this article are to observe the development of traffic waves. Each experiment consists of the following phases:

(i) setup; (ii) evacuation; (iii) initialize; (iv) drive; (v) stop; (vi) conclusion.

During each phase, the following items are performed.

(i) Setup: Vehicles are distributed equally according to the spacing of their front-left tire. Drivers are individually instructed to turn on their in-vehicle recorders. Additional driver instructions (if any) are delivered to individual drivers through the window.

(ii) Evacuation: The central camera is switched on. All research team personnel evacuate the track.

(iii) Initialize: An air horn sounds to instruct all drivers to switch gears from Park to Drive, without moving.

(iv) Drive: An air horn sounds, to instruct all drivers to begin driving.

(v) Stop: An air horn sounds, instructing drivers to come to a safe stop and switch gears into Park.

(vi) Conclusion: Experiment personnel enter the track after all vehicles have stopped. Drivers are individually instructed to turn off their in-vehicle recorders. The central camera is switched off. 


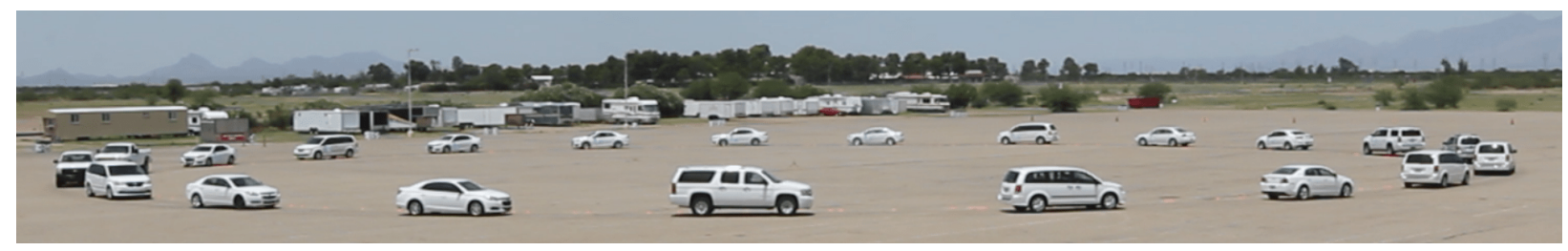

(a) Vehicles driving with an initial uniform spacing.

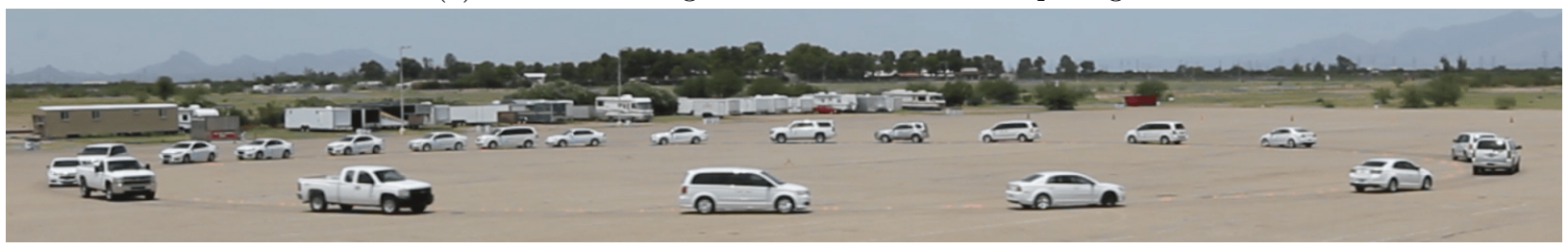

(b) Vehicles driving with a non-uniform spacing, showing a traffic jam.

Figure 1: An overview of the experimental setup for Experiment $\mathrm{H}$.

\subsection{Experimental densities}

This dataset provides complete vehicle trajectories and vehicle performance data for eight experiments that ranged between 19 and 22 vehicles on the $260 \mathrm{~m}$ track. Before testing began, all drivers are given a safety briefing and driving instruction set I.

In order to identify the effect of traffic density on the development of traffic waves, Experiments A and $\mathrm{B}$ are conducted with 20 vehicles. This is increased to 22 vehicles in Experiment C, while not changing the track diameter. In order to explore density space more thoroughly, 21 vehicles are used in Experiment D, and 19 vehicles in Experiment E.

The effect of driver instructions on the development of oscillatory traffic is measured in Experiments F, G, and H, with 19, 21 and 22 vehicles, respectively. For these experiments, drivers are given instruction set II. Experiments F, G, and $\mathrm{H}$ are also used to test the extent to which a single vehicle can alter the traffic state if it drives differently from the remaining vehicles. This is done by changing the driving instructions of a single vehicle during the test via radio communication. The instruction provided to the driver is to maintain a specified target speed, unless the distance and relative velocity to the vehicle ahead require a lower speed. The time at which this instruction is given is referred to as $T_{c m d}$.

\subsection{OBD-II and panoramic video data collection}

Each vehicle in the experiment is instrumented with an on-board diagnostics (OBD-II) scanner to collect vehicle data during the experiments. All vehicles sold in the United States after 1996 are required to provide vehicle performance data from the engine control unit (ECU) via an OBD-II port. The OBD-II data are logged using a ScanTool OBDLink LX scanner. The data provided through the OBD-II port include vehicle dynamics data such as engine speed, vehicle speed, and fuel rate as well as diagnostics data such as sensor voltage of a variety of vehicle sensors, which can be used to identify vehicle malfunction. While the OBD-II data format is standardized, due to different vehicle configurations and vehicle ages, not all vehicles report the same data through OBD-II. All speed and fuel rate data collected from each vehicle are made available at 61 .

A VSN Mobile V360 panoramic video camera is used to record the motion of the vehicles, and is located at the center of the track. It has $360^{\circ}$ horizontal field of view and $60^{\circ}$ vertical field of view, recording at a resolution of $3840 \times 640$ pixels at $30 \mathrm{~Hz}$. The high spatial and temporal resolution of the panoramic camera enables precise tracking of the dynamics of the vehicles in the scene when suitable image processing algorithms are applied, described next. 


\section{Data processing}

Tracking moving objects in a sequence of images (frames) is a well established field in computer vision 65, 66, 67. The term tacking refers to the process of inferring the motion of one or multiple objects in a sequence of images [65. Historically there have been two major categories of tracking techniques based on nonparametric and parametric strategies [65]. Recently, a third class of methods based on deep neural networks have also been proposed.

Nonparametric tracking methods require few assumptions on the underlying dynamics of the object to be tracked. One popular nonparametric method, known as tracking by detection, generates the trajectory of an object in a two-step approach. First the object position is identified in each frame, and then the positions are linked using a proximity criteria to produce the full trajectory. Another category of nonparametric methods are referred to as tracking by matching. These methods reconstruct the trajectory of an object by matching a pixel representation of that object in one frame to the next, iterating over the full time span of the video. The tracking method used in this article is a variant of tracking by matching.

The second major category of tracking algorithms is parametric tracking, which exploits the underlying dynamical model to track the motion of objects. When the dynamical model of the object to be tracked is linear, Kalman filters are often applied. Otherwise, nonlinear filters such as particle filters are chosen for the purpose of object tracking [65].

A third group of methods have recently emerged with the rising popularity of neural networks in artificial intelligence research. As an early example in 2013, stacked denoising autoencoders has been used for visual tracking [68. More recently, convolutional neural networks have been re-purposed for target tracking [69]. Moreover, an end-to-end tracking method based on recurrent neural networks has also been developed [70.

The aforementioned tracking methods have been widely applied in the field of transportation research. Within the category of nonparametric tracking, examples of tracking by detection can be found in 71, 72. Tracking by matching methods are applied in [73, 74. Within the category of parametric tracking, applications of Kalman filtering in visual tracking can be seen in [19, 75]. The use of particle filtering in transportation tracking applications can be found in [76, 77. Furthermore, the application of neural networks for vehicle tracking is studied in [78, 79]. In addition, comprehensive surveys of computer vision algorithms in transportation research can be found in 80, 81.

The main challenge for tracking vehicles in the experimental setting described in Section 2 is that the vehicles may completely stop in the presence of stop-and-go waves. Because many nonparametric methods [71, 82] use the motion of the object to be tracked to identify the object from the background, these methods fail when the object no longer moves. Parametric methods circumvent this problem by using additional information obtained from the dynamical model (e.g., as in the Kalman filtering methods) to localize the object even when it has stopped moving. The accuracy of this approach hinges on the quality of the model specified for the object being tracked.

The tracking by matching approach, with suitable modification to handle stationary vehicles, is chosen in this work for its simplicity and effectiveness. It does not require knowledge about the underlying model of the object dynamics (in contrast to the parametric methods), and it does not require a large training dataset (needed in supervised methods such as many neural networks applications). Although some steps the algorithm such as background subtraction and estimation are computationally intensive, it delivers highly accurate positional estimates and it does not need to run online during the experiments.

We briefly describe the general process to extract the vehicle trajectories from the camera data, which can be split into four major steps: (i) background estimation and subtraction, (ii) object identification, (iii) object tracking, and $(i v)$ noise reduction. Given a panoramic video containing vehicles to be tracked and a nearly static background, each pixel in each frame is labeled as either moving or stationary. Moving pixels often correspond to vehicles and are unlikely to belong to the background scenery, while stationary pixels may belong to the background or to a stationary vehicle. Over a time interval containing multiple frames, an estimate of the complete background scene for the time interval can be constructed by taking a simple median (over time) of the color value of each stationary pixel. A high quality estimate of the background is obtained from the median provided that fewer than half of the frames contain stationary vehicles, which is always true in our experiments. 
Vehicle trajectories are reconstructed from the video by identifying the location of each vehicle throughout the video. First a template of each vehicle is constructed that can be tracked. The background scene is compared to a candidate frame in the video to identify pixels that do not "match" the value in the background scene. These non-matching pixels are identified as belonging to the foreground (i.e., to a vehicle), independent of whether the vehicle is moving or stationary. These foreground pixels are then grouped into vehicles using a density-based clustering algorithm, which is further refined to define a high quality template (i.e., a picture) of the vehicle. After the template is defined, it is used to be re-identify the vehicle in subsequent frames, allowing a trajectory for that vehicle to be constructed. Since the track radius is known a priori, the position of each vehicle in pixel space can be converted to a location on the track via straightforward geometry. The trajectories are further refined using a spline smoothing algorithm with outlier detection, to reduce the effects of small tracking errors and the quantization error induced by the resolution of the camera. These steps are explained in detail below.

\subsection{Background estimation and subtraction}

In this step, an estimate of the background is obtained over a sequence of frames of the video. The process of estimating the background is complicated by the facts that $(i)$ the vehicles occlude the background scene, (ii) occasionally vehicles stop (i.e., in a stop-and-go wave), making them difficult to differentiate from the stationary background using motion-based methods, and ( $\mathrm{iii}$ ) occasionally the background changes or moves (e.g., due to changes in light intensity or the motion of clouds). Consequently, we adopt a strategy that first detects sufficiently stationary pixels in each frame (described in detail below), and we estimate the background as the median pixel value over the set of images. For an individual pixel location, the median of the non-moving pixels provides a reasonable estimate of the background pixel value as long as the majority of frames in which the pixel is stationary do not correspond to a vehicle. This condition is met in our experiments by choosing a time horizon to estimate the background which is sufficiently large relative to the duration over which vehicles are stopped in the stop-and-go wave.

Precisely, the background identification proceeds as follows. Consider a video composed of a sequence of images indexed by $t \in \mathbb{Z}_{+}$, where each image is composed of a matrix of pixels. Denote $V(x, y, t)$ as the value of the pixel in frame $t$ located at $(x, y) \in\left\{1, \ldots, x_{\max }\right\} \times\left\{1, \ldots, y_{\max }\right\}=: \mathcal{X} \times \mathcal{Y}$ in the scene. The parameters $x_{\max }$ and $y_{\max }$ denote the horizontal and vertical resolution of the camera, respectively. For RGB video, $V(x, y, t)$ is a vector of dimension three, with each color channel (red, green, and blue) taking values in $\{0, \ldots, 255\}$. For each pixel $(x, y)$, we determine whether the pixel corresponds to a moving object between frame $t$ and $t+1$ using a standard implementation of dense optical flow [83, which is a computer vision algorithm designed to detect the apparent motion of pixels across two frames. We apply dense optical flow to all consecutive image pairs between $t$ and $t+\Delta t$. The output of the algorithm is an index set for each pixel $\mathcal{S}(x, y) \subseteq\{t, \ldots, t+\Delta t\}$, which contains the frames in which pixel $(x, y)$ is identified to be stationary. Finally, the RGB background corresponding to pixel $(x, y)$ is denoted $B(x, y)$ and computed as

$$
B(x, y)=\operatorname{median}\{V(x, y, \tau) \mid \tau \in \mathcal{S}(x, y)\}
$$

where the median is applied channel-wise.

For practical implementations such as the experiments presented in this work, the estimated background image needs to be slowly updated over time to account for slowly varying changes such as light intensity. In our present implementation, the background is recomputed every 15 seconds. Figure 2 layer 1 shows the estimated static background within first 15 seconds of the Experiment A, as compared to Figure 2 layer 2 which shows the first frame in the corresponding 15 -second time interval, including the vehicles. It is clear from the images that foreground vehicles are completely removed from the estimated background.

After the background is defined, foreground pixels corresponding to vehicles can be identified, independent of whether the vehicle is moving or not, as those with a sufficiently different value from the background. Precisely, for each frame $t$, we compute the foreground pixel set as

$$
\mathcal{F}(t)=\left\{(x, y) \in \mathcal{X} \times \mathcal{Y} \mid \quad\|V(x, y, t)-B(x, y)\|_{2} \geq \gamma\right\}
$$




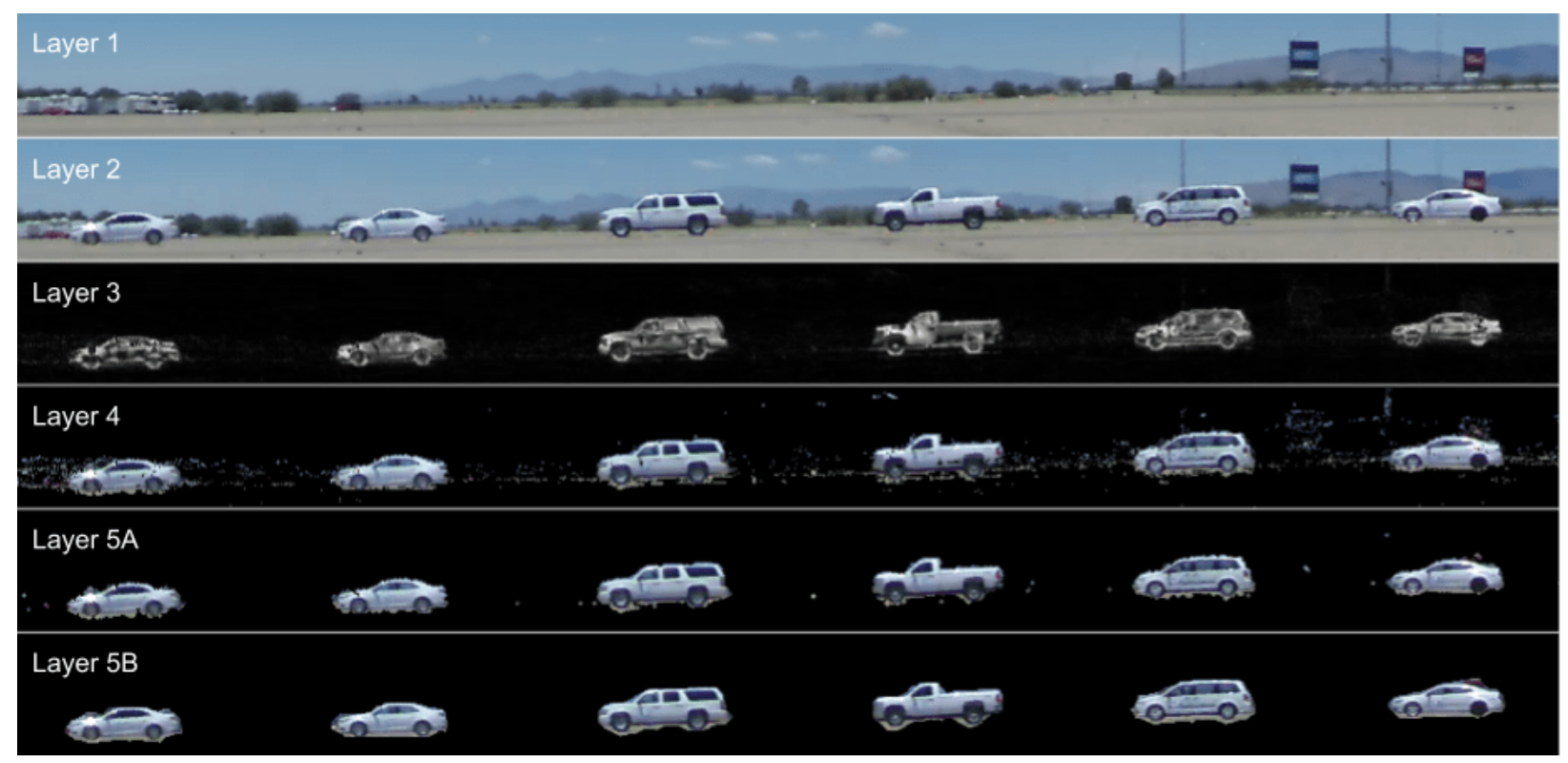

Figure 2: Step-by-step visualization of background subtraction methods. Layer 1 shows a section of the estimated background within the first 15-second time interval in Experiment A. Layer 2 is the first frame of that time interval. Layer 3 is the square distance between layer 1 and layer 2. Layer 4 is the remaining foreground after removing pixels at locations that have square distance under 14. Layer $5 A$ is the refined foreground after applying morphological transformation on layer 4 . Layer $5 B$ is the refined foreground after removing noise pixels outside the alpha complex of every vehicle from layer 4 .

where $\gamma$ is a difference threshold below which the pixel value of the video is assumed to match the background. An example of a set of foreground pixels under the threshold parameter $\gamma=48$ is shown in Figure 2 layer 4 , which shows the vehicles are clearly identified as part of the foreground, along with some additional non-vehicle pixels (noise). In our testing, pixels corresponding to vehicles are identified for a range of $\gamma$ values (e.g., $48 \pm 25 \%$ ), and the threshold choice is not too sensitive.

The foreground set $\mathcal{F}$ contains all pixels estimated as belonging to vehicles, which needs further refinement to eliminate the noise. For example, isolated foreground pixels are unlikely to correspond to vehicles, which typically occupy hundreds of pixels in our implementation. Two standard morphological transformations (i.e., erosion and dilation, see 84 for more detail) are applied, which act as filters to reduce the noise in the foreground pixel set. The refined foreground after applying these denoising steps is shown in Figure 2 layer $5 \mathrm{~A}$, where the noisy pixels are substantially reduced in number.

\subsection{Object identification}

After the background has been subtracted (i.e., pixel-wise and channel-wise subtraction) from every frame, we proceed next to construct a template of each vehicle which can be tracked from one background subtracted frame to the next. A high quality template of each vehicle is constructed on a single frame by first clustering the foreground pixels into vehicles, and then enhancing each vehicle cluster, e.g., by filling in holes. We note that keeping the vehicle template static works the best (compared to allowing the template to be adjusted as time progresses) in our experience, because it prevents the templates from being polluted by random noise. The quality of tracking can degrade substantially if the quality of input template is not maintained.

The construction of the vehicle template via clustering relies on the fact that (in the present experimental setup) the foreground pixels from the same vehicle have smaller Euclidean distance to the pixels corresponding to the same vehicle, compared to pixels from different vehicles. Consequently, a density-based clustering algorithm can effectively group those foreground pixels into clusters. An off-the-shelf unsupervised clustering algorithm known as density-based spatial clustering of applications with noise (DBSCAN) [85] is applied, 

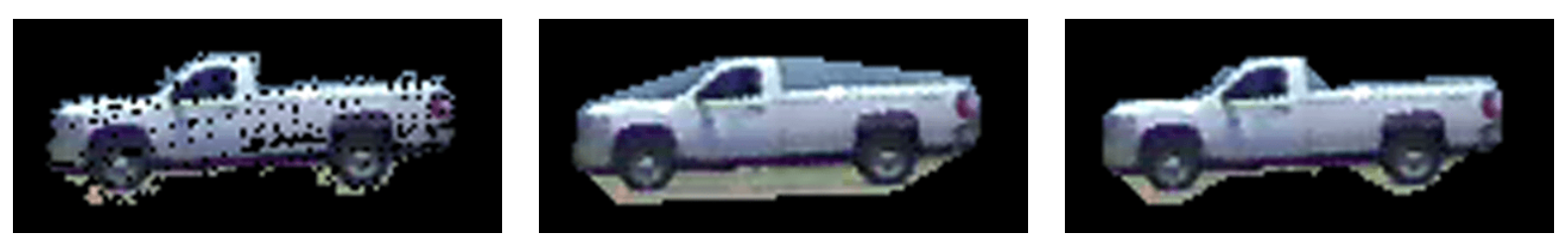

(a) Estimated vehicle template using (b) Vehicle template using the convex (c) Estimated template computed DBSCAN, with holes due to errors in hull of the cluster in (a). Note this is with the alpha complex. the background subtraction step. an intermediate result.

Figure 3: Template construction of a pickup truck in Experiment A.

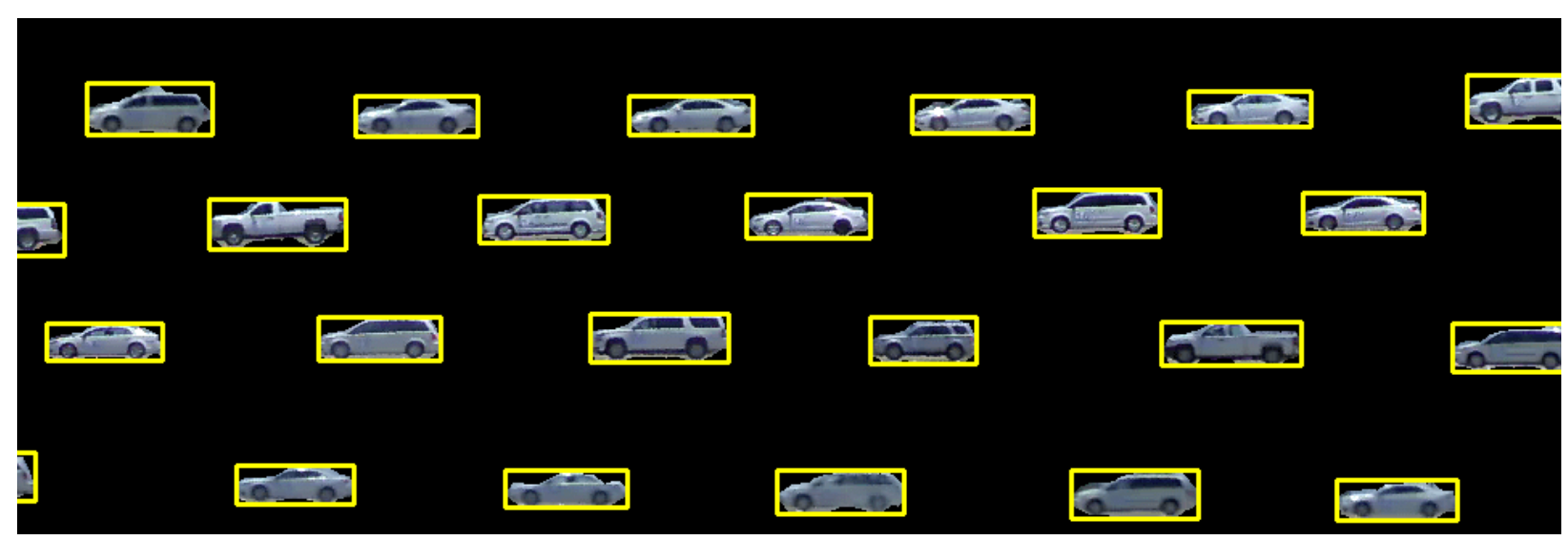

Figure 4: Templates of all vehicles in Experiment A. The template of each vehicle is enclosed by a yellow bounding box, and shows the front and rear bumper locations are also accurately determined (relevant for measuring vehicle gaps).

with a small transformation to the input video data to account for the periodicity of the image recorded by the panoramic camera (i.e., the fact that pixels near $x_{0}$ and $x=x_{\max }$ can correspond to the same vehicle).

The periodicity is addressed transforming the image into an annulus where the beginning and the end of the original image frame are connected, so that DBSCAN can be directly applied. The clustering in DBSCAN is controlled through two parameters corresponding to $(i)$ the maximum nearest-neighbor distance of points within the same cluster $d_{\max }$, (ii) the minimum cluster size $n_{\min }$. In the present work, we set $d_{\max }$ corresponding to 2.5 pixels and $n_{\min }=15$ points. These parameters are observed to generalize well across the various experiments. Finally, the pixel clusters in polar coordinates are mapped back to the Cartesian coordinates for further analysis.

Each cluster identified in DBSCAN is converted into a vehicle template by construction of the alpha complex of the cluster. The the cluster alpha complex results in a possibly non-convex shape to define the vehicle, and can be computed via Delaunay triangulation of the point set [86]. Any missing pixels in the interior of the alpha complex are defined as belonging to the vehicle template, and consequently it "fills in" missing pixels in the template. An example of a cluster from DBSCAN, its convex hull, and the alpha complex are shown in Figure 3. The templates of all vehicles in Experiment A is shown in Figure 4.

\subsection{Object tracking}

Object tracking in this application is the procedure to determine the locations of the vehicles over time. This objective can be achieved by means of frame-by-frame matching of the vehicle template to the foreground image. More precisely, the vehicle template is matched to a transformed image in which the background image has been subtracted and the resulting foreground noise is reduced using morphological operations. In the object tracking step, the RGB images are converted to a scalar greyscale quantity, so that standard implementation libraries (i.e., OpenCV [87]) can be used directly. The implementation details of the technique are as follows. 


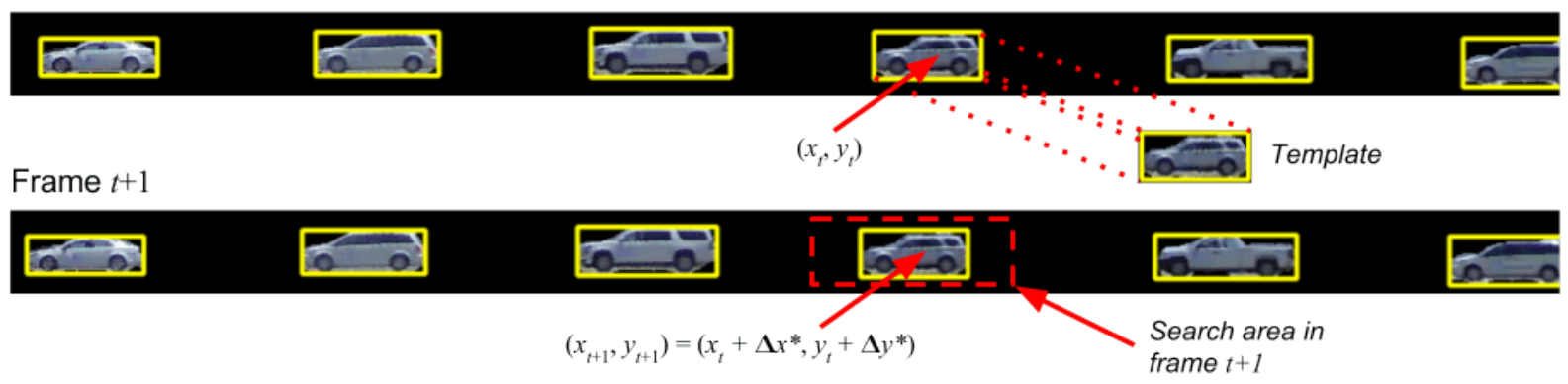

Figure 5: Illustration of the tracking algorithm. For every vehicle in every frame, a small neighborhood is selected based on the location of that vehicle in the previous frame. The template is used to locate the current position of the vehicle via convolution.

Let $\mathcal{T} \subseteq \mathcal{X} \times \mathcal{Y}$ be the union of the pixels defining the vehicle and the black pixels within the vehicle's smallest bounding box. Suppose $T(x, y)$ is a zero-average template of the vehicle constructed in the initial frame $t_{0}$, i.e.,

$$
T(x, y)=\left.V\left(\tilde{x}, \tilde{y}, t_{0}\right)\right|_{(\tilde{x}, \tilde{y}) \in \mathcal{T}}-\frac{1}{|\mathcal{T}|} \sum_{(\tilde{x}, \tilde{y}) \in \mathcal{T}} V\left(\tilde{x}, \tilde{y}, t_{0}\right)
$$

In practice, the template is also constructed on frame $t_{0}=0$ unless otherwise specified.

Let $I(x, y, t)$ be a zero-averaged image over an area $\mathcal{I}(t)=\{(x+\Delta x, y+\Delta y)|(x, y) \in \mathcal{T},| \Delta x|\leq 10,| \Delta y \leq$ $2 \mid\}$ at time $t$ :

$$
I(x, y, t)=\left.V(\tilde{x}, \tilde{y}, t)\right|_{(\tilde{x}, \tilde{y}) \in \mathcal{I}(t)}-\frac{1}{|\mathcal{I}(t)|} \sum_{(\tilde{x}, \tilde{y}) \in \mathcal{I}(t)} V(\tilde{x}, \tilde{y}, t)
$$

We estimate the current location of the vehicle by its shift $\Delta x$ and $\Delta y$ using a zero-centered cross correlation function:

$$
\left(\Delta x^{*}, \Delta y^{*}\right)=\arg \max _{\Delta x, \Delta y} \sum_{(x, y) \in \mathcal{T}} I(x+\Delta x, y+\Delta y, t) \cdot T(x, y),
$$

which is more robust than Euclidean distance measures for noisy scenes 88 .

To avoid mismatching an adjacent vehicle, we search the template only in a small neighborhood of the vehicle location identified in the previous frame, as illustrated in Figure 5. Specifically, we restrict $|\Delta x| \leq 10$ and $|\Delta y| \leq 2$, based on physical bounds on the vehicle velocities under small tracking errors (e.g., the vehicle speed is non-negative, but we allow a small search window backwards to allow for small negative tracking corrections).

Finally, the vehicle trajectories are mapped from video units into physical units. This involves converting time from frames into seconds and converting distance data from pixels into meters. Time conversion can be simply done using the fixed frame rate of $30 \mathrm{fps}$. To convert the distance measures, one can exploit the fact that the track is circular with a 260-meter circumference (at the center line of the vehicles). Hence, dividing 260 meters by 3840 pixels (the width of a frame), one obtains the conversion factor of $\frac{260}{3840} \approx 0.0915$ meters/pixel.

\subsection{Noise reduction}

To improve the quality of the data generated from the tracking algorithm, a basis spline (B-spline) noise reduction method is applied. We first describe the major types of noise observed in the dataset, and then describe the smoothing technique used to reduce the noise.

There are two primary sources of noise in the trajectory data produced from the image processing algorithm described above: i) quantization errors and ii) burst noise. Quantization errors arise from the 
limited resolution of the panoramic camera. As a result, the distance measure is quantized to pixels, and time only advances in discrete steps of $\frac{1}{\text { framerate }}=\frac{1}{30}$ second. If left unsmoothed, this limited spatial and temporal resolution would restrict velocity estimates to integer multiples of $30 \mathrm{px} / \mathrm{s}$, or $2.03 \mathrm{~m} / \mathrm{s}$, and acceleration estimates to integer multiples of $900 \mathrm{px} / \mathrm{s}^{2}$, or $60.9 \mathrm{~m} / \mathrm{s}^{2}$, assuming finite difference estimates using directly adjacent frames are used. Burst noise is produced when irregular events occur during the experiments. The tracking algorithm is built upon a set of assumptions, for example, the background subtraction step assumes stable lighting conditions. While those assumptions hold true most of the time, they are occasionally violated due to changing light conditions resulting from fast-moving clouds, or mirror-like reflectivity (i.e., specularity) of the vehicle paint. In these cases, one may observe burst noise in the raw vehicle trajectories.

To reduce the noise described above, a fourth-order weighted B-spline smoother is applied to reconstructed trajectories, which is able to substantially reduce the noise [89, 90]. The choice of a fourth-order B-spline allows for smoothness in the acceleration estimates of the vehicle trajectories. Concretely, the spline smoother uses a weighted square loss function, where splines are connected on the boundaries under $C_{3}$ continuity. The weights are chosen such that acceleration estimates obtained from the second derivative of the B-splines are observed to be within physically reasonable bounds. Additionally, a parameter called tolerance (precisely, a limit on the sum of the absolute error between the data points and the spline over an interval) is used to control the length of every individual fitting window. Because a fourth-order spline is uniquely defined by five points, fitting error will likely arise if more than five points are given. Very commonly, such fitting error increases with the length of fitting window. Therefore, one can implicitly control window length by specifying a tolerance value such that the window is as long as possible under the constraint that the fitting error does not exceed the tolerance. The influence of the tolerance parameter on the accuracy of the trajectories is explored in detail in the next section.

\section{Data validation}

The tracking algorithm described in Section 3 is validated both in terms of positional accuracy and in terms of velocity accuracy. Position estimates are compared with a manually labeled dataset, while the velocity data are validated by comparing to high-precision velocity data recorded on the highly instrumented University of Arizona Cognitive and Autonomous Test Vehicle (CAT Vehicle), which is vehicle number 20 in each experiment. The distribution of vehicle accelerations and fuel rate is also presented, due to the lack of validation datasets for these measurements.

\subsection{Position accuracy}

We compare the positions estimated from the image processing algorithm with manually labelled position data. Manual labels are generated through three human annotators using an online annotation tool, LabelMe 91]. The annotators were asked to label the rear bumper location of vehicle 19 and the front bumper location of vehicle 20. One annotator labeled frames at a framerate of $3 \mathrm{~Hz}$, for a total of 900 frames (five minutes) of Experiment A. Two additional annotators were instructed to independently label the first 33 seconds of the experiment at the same frame rate, so that the inter-annotator agreement can be computed for the three annotators. The sample standard deviation of the position labels is $0.05 \mathrm{~m}$ ( 0.75 pixels). Consequently the human annotation data can be used as a precise proxy of the true position of each vehicle.

The position data extracted from the camera are compared on the 900 frame dataset using both the raw and the smoothed camera trajectory data of vehicle 19 and 20. We treat the human annotated position estimates as the true position of the vehicle, from which error residuals and the standard deviation of the error distribution can be computed. The average error of the raw camera position estimates is $-0.04 \mathrm{~m}$, and the standard deviation of the errors is $0.12 \mathrm{~m}$. The average error of the smoothed camera trajectory is less than $0.01 \mathrm{~m}$, and the errors have a standard deviation of $0.11 \mathrm{~m}$. The smoothed signal and the raw signal have similar position error distributions. The error distributions of the raw and noise-reduced position estimates are shown in Figure6 


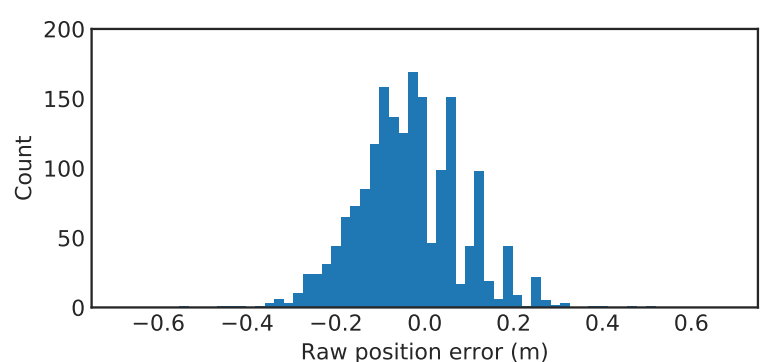

(a) Distribution of the raw position errors with human labeled positions taken as the true positions.

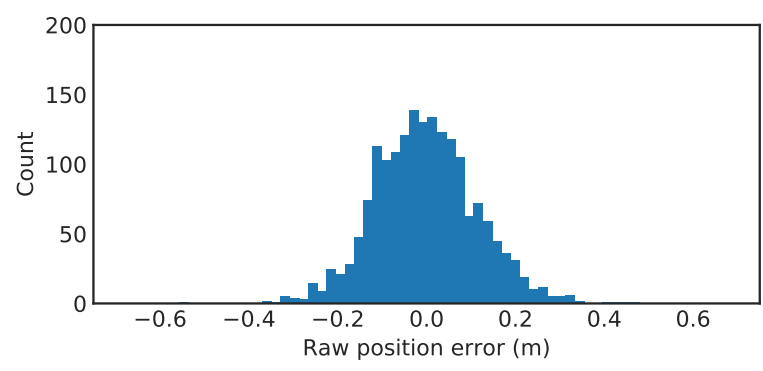

(b) Distribution of the smoothed position errors with human labeled positions taken as the true positions.

Figure 6: Results of validation analysis on the position measurements in Experiment A.
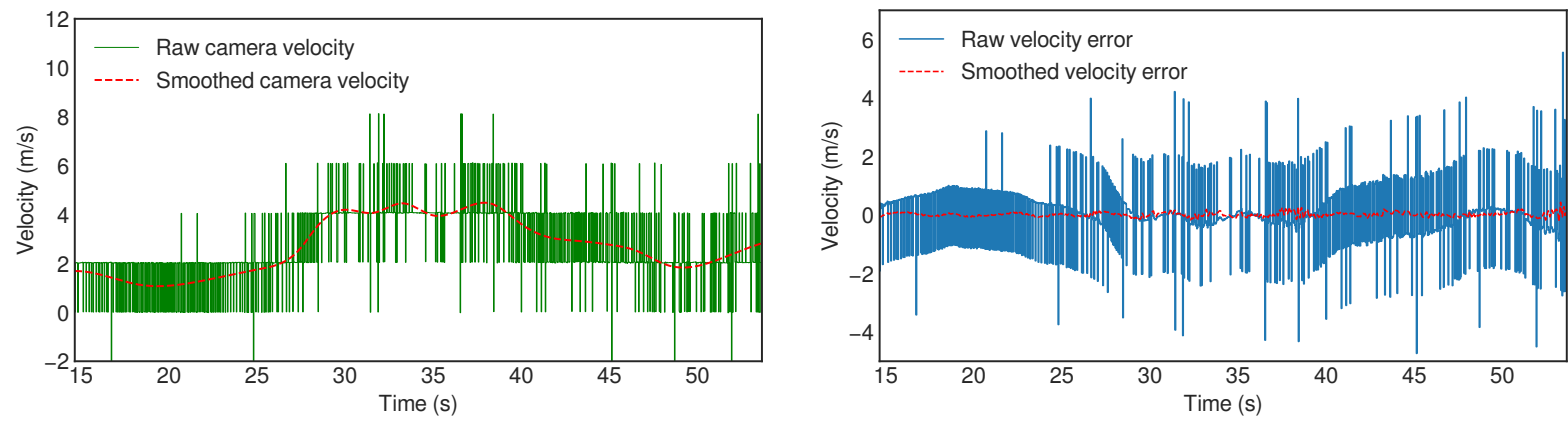

(a) Comparison of velocity signals for vehicle 20 in Exper- (b) Validation of velocity signals for vehicle 20 in Experiment A. The raw camera speed signal (green), quantized iment A. Here the odometry velocity signal is selected as into integer multiples of $2.03 \mathrm{~m} / \mathrm{s}$ oscillates around the the reference. The difference between raw camera velocsmoothed camera velocity signal (red). ity signal and the reference is shown in blue, while the difference between the smoothed velocity signal and the reference displayed in red.

Figure 7: Analysis on B-spline smoothing for noise reduction. The time series plot shows the effects of the B-spline smoother. The residual plot illustrates that the noise reduction method significantly improves the data quality.

\subsection{Velocity accuracy}

The accuracy of the smoothed camera velocity estimate is compared to the velocity recorded from an odometry sensor on vehicle 20 (the highly instrumented CAT Vehicle) in Experiment A. In the following discussion, we treat the odometry data from the CAT Vehicle as the true velocity signal from which errors are computed. We also compare the velocity recorded directly from the ScanTool OBDLink LX OBD-II scanner installed on all vehicles in the experiment (including the CAT Vehicle). Since the odometry-based velocity readings are recorded at $20 \mathrm{~Hz}$, the $30 \mathrm{~Hz}$ camera data are downsampled from $30 \mathrm{~Hz}$ to $20 \mathrm{~Hz}$ for the task of velocity validation.

The difference between the raw camera signal and the smoothed camera signal is illustrated in Figure 7 for Experiment A. The effects of B-spline smoothing is demonstrated in Figure 7a, while the accuracy of the raw and smoothed camera velocity is shown in Figure 7b. In Figure 7a, the raw camera velocity contains clear quantization errors with a step size of about $2.03 \mathrm{~m} / \mathrm{s}$, while the smoothed camera velocity is free of the quantization errors. Irregularly large estimates, or burst noises, in the raw camera velocity such as 8.12 $\mathrm{m} / \mathrm{s}$ and $-2.03 \mathrm{~m} / \mathrm{s}$ are also removed in the smoothed data. As evident in Figure $7 \mathrm{~b}$, the noise reduction technique converts the original noisy measurements to one that is one to two orders of magnitude closer to the odometry velocity. 


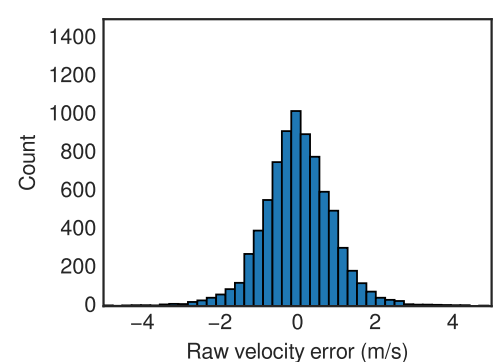

(a) Distribution of difference between raw velocity and odometry velocity.

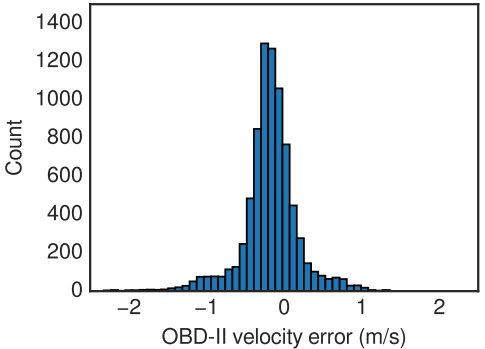

(b) Distribution of difference between (c) Distribution of difference between OBD-II velocity and odometry veloc- smoothed camera velocity and odomity.

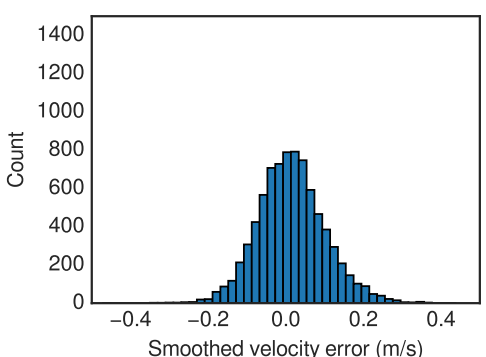
etry velocity.

Figure 8: Results of validation analysis on the speed measurements in Experiment A.

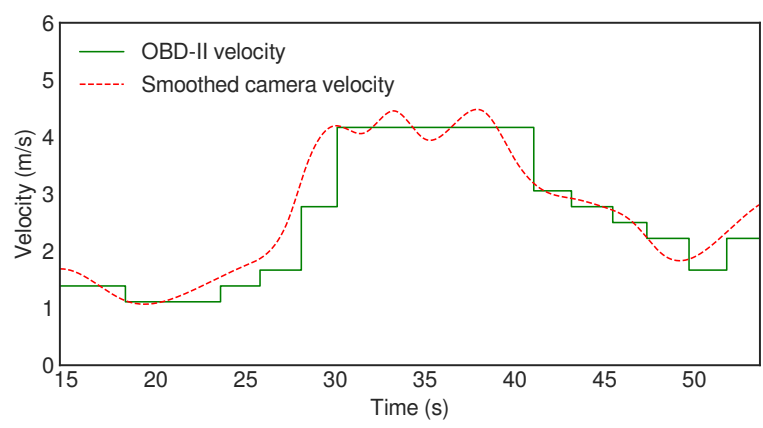

(a) Comparison of velocity signals for vehicle 20 in Ex- (b) Validation of velocity signals for vehicle 20 in Experperiment A. The OBD-II recorded speed signal (green) is iment A. Here the odometry velocity signal is selected quantized in time and space.

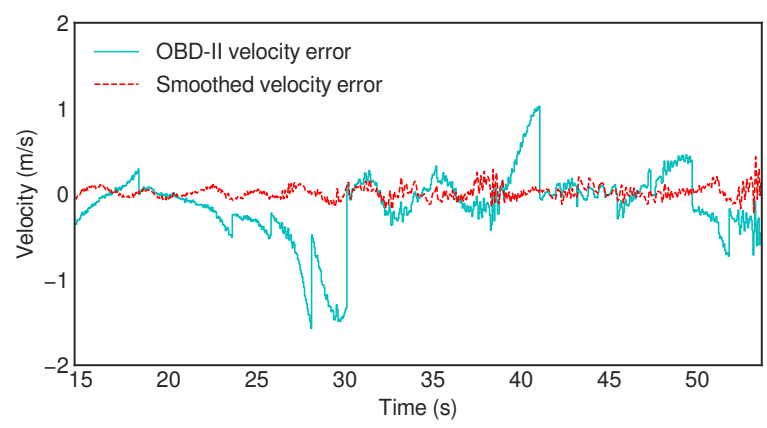

as the reference. The difference between OBD-II velocity signal and the reference is shown in blue, while the difference between the smoothed velocity signal and the reference displayed in red.

Figure 9: Comparison between smoothed camera velocity data and OBD-II velocity data.

In Figure 8 , the error distributions of the raw camera velocity, the OBD-II recorded velocity, and the smoothed camera velocity for vehicle 20 in Experiment A are shown. The smoothed camera velocity error distribution has a standard deviation $(0.09 \mathrm{~m} / \mathrm{s})$ that is an order of magnitude smaller than the raw camera data $(1.17 \mathrm{~m} / \mathrm{s})$, and it is also smaller than the OBD-II velocity error standard deviation $(0.37 \mathrm{~m} / \mathrm{s})$. The surprising finding that the camera speeds are more precise is due to the (undocumented) internal processing of the OBD-II signal that occurs either on the vehicle or in the OBD-II scanner. From Figure 9, we observe that the OBD-II recorded velocity is also quantized, and appears to hold the value of the recorded velocity constant over several seconds. This leads to larger errors than the smoothed camera velocity data.

\subsection{Acceleration and fuel rate distribution}

Due to the lack of other reliable measures against which the collected data can be validated, we show that the distribution of the accelerations and fuel rate data are physically reasonable (e.g., the accelerations lie within the bounds of the physical performance limits of the vehicles). The distribution of acceleration measurements for all vehicles in Experiment A is shown in Figure 10a, and the distribution of fuel rate measurements is shown in Figure 10b. For common commercial vehicles, the magnitude of acceleration is less than $5 \mathrm{~m} / \mathrm{s}^{2}$ and the limit of fuel rate less than $25 \mathrm{l} / \mathrm{h}$ [92, 93], and the collected dataset is shown to 


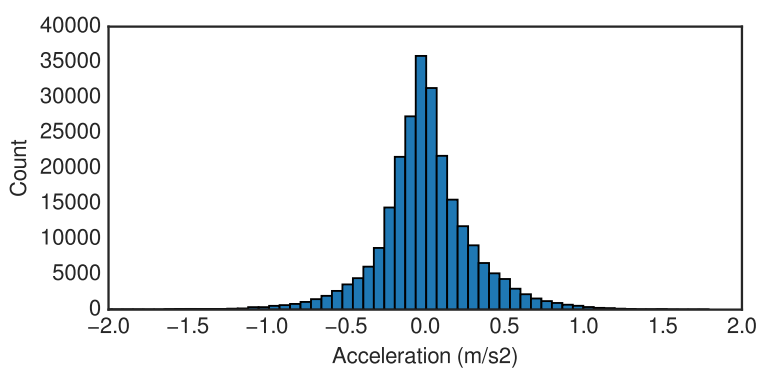

(a) Distribution of acceleration measurements.

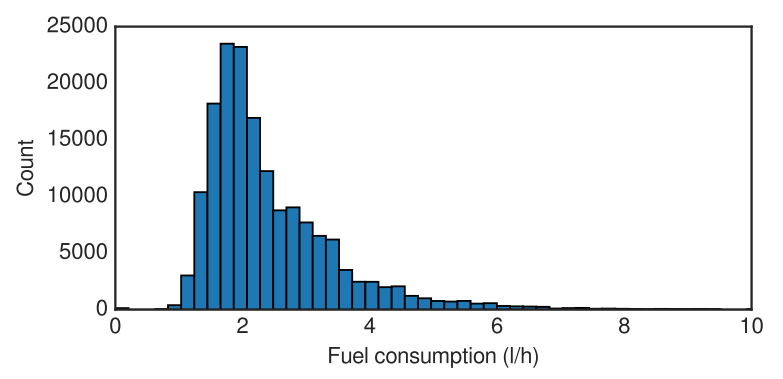

(b) Distribution of fuel consumption rate measurements.

Figure 10: Distributions of acceleration and fuel rate measurements in Experiment A.

comply these limits. In fact, the magnitude of acceleration rarely exceeds $1.5 \mathrm{~m} / \mathrm{s}^{2}$, and the fuel rate rate rarely exceeds $8 \mathrm{l} / \mathrm{h}$. The distributions of acceleration and fuel rate for the other experiments are similar to those of Experiment A, and therefore are not presented in this article. A summary of the upper and lower limits of the acceleration and fuel rate distributions for each experiment is tabulated in Table $1 \mathrm{~b}$.

\subsection{Noise reduction parameters selection}

Note that the results presented above are not too sensitive to the tolerance parameter of the B-spline smoother.Figure 11a shows the result of a sensitivity analysis, which illustrates that any tolerance value between $11 \mathrm{~m}$ and $17 \mathrm{~m}$ produces small error standard deviations in position data and velocity data. Figure $11 \mathrm{~b}$ indicates that the estimation bias in the position and velocity data are also minimized when the tolerance is between $11 \mathrm{~m}$ and $17 \mathrm{~m}$. Consequently the tolerance parameter is set to be $14 \mathrm{~m}$, which lies in the center of the optimal interval. Recall that the tolerance controls the cumulative error between the raw data and the B-spline, and indirectly controls the window (number of points fit with a single spline). When the tolerance is set to $14 \mathrm{~m}$, the window typically contains hundreds or thousands of points.

Moreover, it is shown in Table 1 that the specific choice of the smoother parameter in Experiment A generalizes well to the other experiments. Specifically, we apply the optimal smoother parameter from experiment A to the remaining experiments, which were not used to determine the optimal parameter. Under the threshold choice of $14 \mathrm{~m}$, the maximum (over all tests) mean error is $0.03 \mathrm{~m} / \mathrm{s}$ with a standard deviation of $0.11 \mathrm{~m} / \mathrm{s}$ of smoothed velocity error mean is $0.02 \mathrm{~m} / \mathrm{s}$, and the maximum ranges of smoothed velocity error standard deviation is $0.03 \mathrm{~m} / \mathrm{s}$. Therefore the selected smoother parameters generalize well to other experiments and the high quality velocity estimates are not an artifact of overfitting the tolerance parameter on a single dataset.

\subsection{Data anomalies}

Although the data collected from the experiments presented in this article are largely complete, there are a few anomalies that must be noted. These take the form of missing or erroneous measurements in the OBD-II data.

Concretely, the OBD-II fuel rate data $(\mathrm{l} / \mathrm{h})$ contain missing entries, zero readings, and an inconsistency in sampling rate. OBD-II data is missing from the the following vehicles: Experiment D, vehicles 5 and 6; Experiment E, vehicle 3; Experiment F, vehicle 17; Experiment H, vehicle 4. Zero fuel rate readings are recorded for vehicle 15 in Experiment A for the first four seconds of the experiment. These missing data entries and zero readings are the result of operational errors with the OBD-II scanners during the experiment. Additionally, while the experiments are designed to collect OBD-II data at the maximum sampling rate of $20 \mathrm{~Hz}$, the OBD-II scanner in vehicle 19 collected data at $10 \mathrm{~Hz}$ for all experiments due to an incorrect setting in the OBD-II logger. 


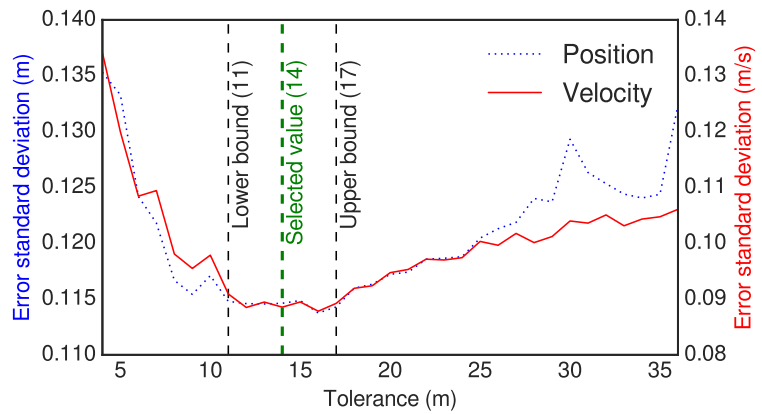

(a) Error standard deviations as a function of the tolerance parameter used in B-spline smoothing.

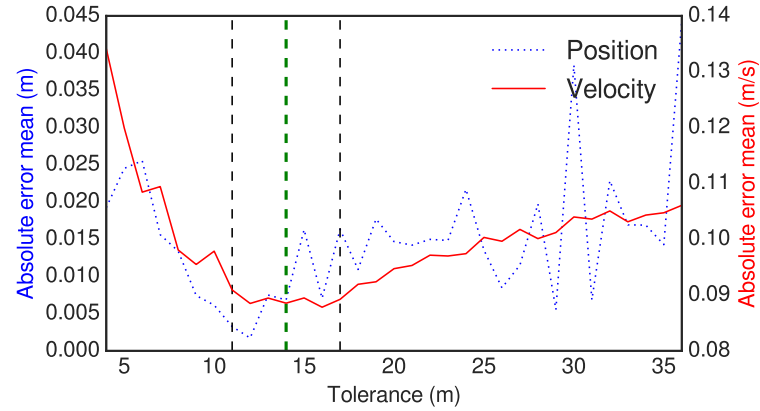

(b) Error standard deviations as a function of the tolerance parameter used in B-spline smoothing.

Figure 11: Results of parameter tuning using the position labels and odometry measurements in Experiment A.

\begin{tabular}{cccc}
\hline $\begin{array}{c}\text { Exp. } \\
\text { No. }\end{array}$ & $\begin{array}{c}\text { Raw velocity } \\
\text { error }(\mathbf{m} / \mathbf{s})\end{array}$ & $\begin{array}{c}\text { OBD-II } \\
\text { velocity error } \\
(\mathbf{m} / \mathbf{s})\end{array}$ & $\begin{array}{c}\text { Smoothed } \\
\text { velocity error } \\
(\mathbf{m} / \mathbf{s})\end{array}$ \\
\hline A & $0.02 \pm 1.17$ & $-0.18 \pm 0.37$ & $0.02 \pm 0.09$ \\
B & $0.02 \pm 0.65$ & $-0.18 \pm 0.32$ & $0.02 \pm 0.08$ \\
C & $0.03 \pm 0.62$ & $-0.16 \pm 0.47$ & $0.03 \pm 0.09$ \\
D & $0.03 \pm 0.64$ & $-0.17 \pm 0.28$ & $0.03 \pm 0.11$ \\
E & $0.02 \pm 0.61$ & $-0.17 \pm 0.45$ & $0.02 \pm 0.09$ \\
F & $0.01 \pm 0.61$ & $-0.23 \pm 0.34$ & $0.01 \pm 0.11$ \\
G & $0.01 \pm 0.61$ & $-0.21 \pm 0.82$ & $0.01 \pm 0.09$ \\
H & $0.02 \pm 0.61$ & $-0.20 \pm 0.57$ & $0.02 \pm 0.08$ \\
\hline
\end{tabular}

(a) Velocity bias \pm one standard deviation are reported.

\begin{tabular}{ccc}
\hline $\begin{array}{c}\text { Exp. } \\
\text { No. }\end{array}$ & $\begin{array}{c}\text { Acce- } \\
\text { leration } \\
\left(\mathbf{m} / \mathbf{s}^{2}\right)\end{array}$ & $\begin{array}{c}\text { Fuel } \\
\text { rate } \\
(\mathbf{l} / \mathbf{h})\end{array}$ \\
\hline A & $-3.17-1.79$ & $0.71^{*}-10.34$ \\
B & $-1.78-2.33$ & $0.89-09.38$ \\
C & $-1.70-1.65$ & $0.90-12.71$ \\
D & $-2.22-1.98$ & $0.77-13.65$ \\
E & $-2.46-1.85$ & $0.88-15.65$ \\
F & $-3.65-3.50$ & $0.76-22.12$ \\
G & $-5.35-4.19$ & $0.88-23.67$ \\
H & $-4.45-4.05$ & $0.86-23.45$ \\
\hline
\end{tabular}

(b) Acceleration and fuel rate ranges.

Table 1: Summary of validation analysis on velocity, acceleration and fuel rate data. The velocity error for is defined to be the difference between the velocity measure of interest $v$ and the odometry velocity readings $v_{\text {odo }}$, i.e., $v-v_{o d o}{ }^{*}$ The abnormal zero fuel rate readings recorded from vehicle 15 at the start of experiment A are excluded from the calculation. 


\begin{tabular}{cccccccc}
\hline $\begin{array}{c}\text { Exp. } \\
\text { mo. }\end{array}$ & $\begin{array}{c}\text { No. of } \\
\text { vehicles }\end{array}$ & $\begin{array}{c}\text { Instru- } \\
\text { ction } \\
\text { set }\end{array}$ & $\begin{array}{c}\bar{v} \pm \sigma_{v} \\
\text { before in- } \\
\text { tervention } \\
(\mathbf{m} / \mathbf{s})\end{array}$ & $\begin{array}{c}\bar{v} \pm \sigma_{v} \\
\text { after inter- } \\
\text { vention } \\
(\mathbf{m} / \mathbf{s})\end{array}$ & $\begin{array}{c}\bar{r} \pm \sigma_{r} \\
\text { before in- } \\
\text { tervention } \\
(\mathbf{l} / \mathbf{h} / \mathbf{v e h})\end{array}$ & $\begin{array}{c}\bar{r} \pm \sigma_{r} \text { after } \\
\text { interven- } \\
\text { tion } \\
(\mathbf{l} / \mathbf{h} / \mathbf{v e h})\end{array}$ & $\begin{array}{c}\text { Duration } \\
\text { (s) }\end{array}$ \\
\hline $\mathrm{A}$ & 20 & I & $3.11 \pm 0.80$ & - & $2.46 \pm 1.07$ & - & 416 \\
$\mathrm{~B}$ & 20 & I & $2.81 \pm 0.69$ & - & $2.36 \pm 0.92$ & - & 442 \\
$\mathrm{C}$ & 22 & I & $2.37 \pm 0.55$ & - & $2.23 \pm 0.80$ & - & 388 \\
$\mathrm{D}$ & 21 & I & $3.15 \pm 0.70$ & - & $2.51 \pm 1.04$ & - & 480 \\
$\mathrm{E}$ & 19 & I & $3.88 \pm 0.91$ & - & $2.62 \pm 1.21$ & - & 333 \\
$\mathrm{~F}$ & 19 & II & $7.68 \pm 0.96$ & $5.79 \pm 1.91$ & $3.90 \pm 2.34$ & $3.58 \pm 2.63$ & 175 \\
$\mathrm{G}$ & 21 & II & $5.27 \pm 2.63$ & $5.81 \pm 1.28$ & $4.21 \pm 3.28$ & $3.67 \pm 2.66$ & 587 \\
$\mathrm{H}$ & 22 & II & $5.07 \pm 2.46$ & $4.51 \pm 1.89$ & $4.14 \pm 3.04$ & $3.47 \pm 2.60$ & 545 \\
\hline
\end{tabular}

Table 2: Summary of experiments conducted. Instruction set I is to "safely follow the vehicle in front as if in rush hour traffic", while instructions II is to "place an emphasis on closing the gap the the vehicle in front." In Experiments $\mathrm{F}$, G, and $\mathrm{H}$, the driver of a single vehicle was instructed intervene by maintaining a constant speed. $\bar{v}$ is the average velocity, $\sigma_{v}$ is the velocity standard deviation, $\bar{r}$ is the average fuel rate, and $\sigma_{r}$ is the fuel rate standard deviation. Note that after the second intervention in Experiment H, the average velocity is $2.35 \pm 1.21 \mathrm{~m} / \mathrm{s}$ and the average fuel rate is $3.33 \pm 2.39 \mathrm{l} / \mathrm{hr} / \mathrm{veh}$.

\section{Experimental results}

A total of eight experiments are conducted and briefly summarized below. The experiments are compared with respect to a number of quantitative measures. Because the track circumference is fixed, a change in the number of vehicles directly changes the density of traffic on the ring. We present the average velocity, which is proportional to the throughput for a fixed number of vehicles on the test. Two sets of driver instructions are also compared to show how the driver behavior influences the traffic flow. To quantify the velocity variability (which is used as a measure of the wave strength), the velocity standard deviation for critical intervals in each test is presented. Precisely, the wave strength is quantified as the standard deviation of the $m$ velocity measurements (per vehicle) from $n$ vehicles over a time interval. Let $v_{j}^{i}$ denote the $j^{\text {th }}$ velocity measurement from vehicle $i$. The velocity standard deviation is computed as

$$
\sigma_{v}=\left(\frac{1}{m n-1} \sum_{j=1}^{m} \sum_{i=1}^{n}\left(v_{j}^{i}-\bar{v}\right)^{2}\right)^{\frac{1}{2}}
$$

where $\bar{v}$ is the average velocity defined by:

$$
\bar{v}=\frac{1}{m n} \sum_{j=1}^{m} \sum_{i=1}^{n} v_{j}^{i} .
$$

Similarly, the average fuel rate $\bar{r}$ and fuel rate standard deviation $\sigma_{r}$ is also computed for each test.

For experiments A-E, the quantities are computed over the full experiment duration minus an initial period where the vehicles are accelerating from rest. In Experiments $\mathrm{F}-\mathrm{H}$, a single vehicle is commanded to reduce the speed (Experiment $F$ ) or maintain a target speed ( $G$ and $H$ ) to understand the influence of a single vehicle in the stream. In these tests, the relevant quantities are computed both prior to the intervention and after the intervention.

\subsection{Quantitative descriptions of each experiment}

The trajectory, velocity profile, acceleration profile, and fuel rate for each vehicle in each experiment is presented in Figure A.1 through Figure A.8 in the Appendix. A brief description of each experiment is provided below, and a summary of the data collected is provided in Table 2 . 
In Experiment A, 20 vehicles are used on the $260 \mathrm{~m}$ track, and instruction set $\mathrm{I}$ is given to the drivers. The vehicle trajectories are shown in Figure A.1 and small traffic waves are observed. The average speed is $3.11 \mathrm{~m} / \mathrm{s}$ with a velocity standard deviation of $0.80 \mathrm{~m} / \mathrm{s}$, and the average fuel rate is measured to be 2.46 $\mathrm{l} / \mathrm{h} /$ veh. The experiment is ended after 416 seconds.

Experiment B is conducted with the same vehicle density and set of driver instructions as Experiment A. The resulting traffic is slightly slower and less oscillatory than in Experiment A. The waves are shown in Figure A.2, where the average speed is $2.81 \mathrm{~m} / \mathrm{s}$, velocity standard deviation is $0.69 \mathrm{~m} / \mathrm{s}$. The fuel rate in this experiment is $2.36 \mathrm{l} / \mathrm{h} / \mathrm{veh}$. This experiment is ended after 442 seconds.

The vehicle density is increased from 20 vehicles on the $260 \mathrm{~m}$ track to 22 vehicles in Experiment C, while maintaining the same driver instruction set as before. The resulting traffic is both slower and has lower velocity standard deviation than both Experiments A and B. The traffic in Experiment C (shown in Figure A.3 has an average velocity of $2.37 \mathrm{~m} / \mathrm{s}$, and a velocity standard deviation of $0.55 \mathrm{~m} / \mathrm{s}$. The average fuel rate in this experiment is $2.23 \mathrm{l} / \mathrm{h} / \mathrm{veh}$, which is also lower than both Experiment $\mathrm{A}$ and $\mathrm{B}$ where the vehicle density was lower. The experiment is ended after 388 seconds.

Experiment D is conducted with 21 vehicles, and the average speed and velocity standard deviation increase with respect to experiment $\mathrm{C}$. The average speed is $3.15 \mathrm{~m} / \mathrm{s}$ while the velocity standard deviation is $0.70 \mathrm{~m} / \mathrm{s}$. In this experiment, a higher fuel rate than in the previous experiment of $2.51 \mathrm{l} / \mathrm{h} / \mathrm{veh}$ is also observed. The resulting vehicle trajectories can be seen in Figure A.4. The experiment is ended after 480 seconds.

The last experiment conducted with Instruction set I is Experiment E. This experiment has the lowest vehicle density with 19 vehicles on the track. The result is faster moving traffic with stronger waves as seen in Figure A.5. The average velocity in this experiment is $3.88 \mathrm{~m} / \mathrm{s}$ and the velocity standard deviation is $0.91 \mathrm{~m} / \mathrm{s}$. This is the fastest and most oscillatory traffic observed when instruction set I is used. This also results in Experiment $\mathrm{E}$ having the highest fuel rate of all experiments that use instruction set I, with a fuel rate of $2.62 \mathrm{l} / \mathrm{h} / \mathrm{veh}$. The experiment is ended after 333 seconds.

To contrast the effect on the development of traffic waves of the instructions given to drivers, Experiment $\mathrm{F}$ is also conducted with 19 vehicles on the track. However, different from Experiment E, in Experiment $\mathrm{F}$, drivers are given instruction set II. In this case, a larger velocity standard deviation is observed compared to the experiments in which instruction set I was given, as seen in Figure A.6. The average velocity over the first 59 seconds of the experiment is $7.68 \mathrm{~m} / \mathrm{s}$, nearly twice the velocity in any of the previous experiments. Due to the substantial speed increase, the driver of the CAT Vehicle (vehicle 20) is told via radio to slow down and maintain a constant speed as a safety precaution. The result of this is a slowdown of all vehicles on the track, as seen in Figure A.6. The average velocity standard deviation over the first 59 seconds is $0.96 \mathrm{~m} / \mathrm{s}$ and the fuel rate over the same interval is observed to be $3.90 \mathrm{l} / \mathrm{h} / \mathrm{veh}$. The experiment is ended after only 175 seconds. After the conclusion of Experiment F, a safety check is performed with all drivers to address any potential safety concerns due to the higher speed. Due to the significant impact of the intervention by a single vehicle, and the short duration of the experiment, the results are presented here, but not used for further analysis.

Experiment $\mathrm{G}$ is conducted with 21 vehicles on the track, using instruction set II. The resulting traffic waves are larger than those in Experiment F, and significantly more pronounced than the oscillations observed in Experiments A through E. The average speed is $5.27 \mathrm{~m} / \mathrm{s}$ (velocity standard deviation of 2.63 $\mathrm{m} / \mathrm{s}$ ) and the fuel rate is $4.21 \mathrm{l} / \mathrm{h} /$ veh over the first 312 seconds of the experiment. At this point, the driver of the CAT Vehicle is again instructed to drive with a constant speed, this time of $6.26 \mathrm{~m} / \mathrm{s}$ (specifically, the command to drive at $14 \mathrm{mph}$ was given, since a US vehicle was used). However, due to the limits in the precision of human driving behavior, this speed is not strictly maintained by the CAT Vehicle. After the intervention the velocity standard deviation decreases by more than a half to $1.36 \mathrm{~m} / \mathrm{s}$. This indicates that a single vehicle may be able to reduce the speed variability of the flow. This is seen in Figure A.7. The experiment is ended after 587 seconds.

To further explore the difference in wave development under instruction sets I and II, Experiment $\mathrm{H}$ is conducted with instruction set II and 22 vehicles on the track. The average speed in the first 191 seconds of the experiment is $5.07 \mathrm{~m} / \mathrm{s}$, the velocity standard deviation is $2.46 \mathrm{~m} / \mathrm{s}$, and the average fuel rate is 4.14 l/hr/veh. As with Experiment G compared to D, Experiment H compared to C has significantly larger 
waves, and a significantly higher fuel rate due to the change in the instructions. Again as in Experiment G, the driver of a single vehicle is instructed to maintain a constant after some time. In the case of Experiment $\mathrm{H}$, this occurs twice: first after 191 seconds when the driver of the CAT Vehicle is instructed to drive at 5.36 $\mathrm{m} / \mathrm{s}(12 \mathrm{mph})$, and again after 411 seconds, when the driver of the CAT Vehicle is instructed to reduce the speed to $4.47 \mathrm{~m} / \mathrm{s}(10 \mathrm{mph})$. The influence of this intervention on the vehicle speeds and vehicle trajectories is observed in Figure A.8. The velocity standard deviation while the CAT Vehicle is intervening is lower than in the first portion of the experiment. Experiment $\mathrm{H}$ is ended after 545 seconds.

It is worth noting that no experiment with 20 vehicles and instruction set II is conducted due to time constraints on the day of testing.

\subsection{Wave strength and fuel rate}

The data presented in Table 2 summarize the results observed in the eight experiments. The general trends observed in the data are outlined as follows: $(i)$ the instructions given to drivers make a significant difference on the magnitude of waves; $(i i)$ over the range of densities explored, lower density results in stronger waves (measured as the instantaneous velocity standard deviation of all vehicles averaged over the duration of the experiment) when instruction set I was given. A stronger wave is observed at a higher density when instruction set II is given; and (iii) stronger waves result in a higher fuel rate.

The velocity standard deviation depends strongly on the instructions given to the drivers. This is seen in Figure 12a, where the blue squares represent experiment runs in which drivers were given instruction set I and instructed to, "follow the vehicle in front and drive as if you were in rush hour traffic," while the red triangles are trials where drivers were given instruction set II and told to "place an emphasis on closing the gap with the vehicle in front."

The intensity of traffic waves observed also depends on the density of vehicles on the road. In this case, since the track length remains unchanged, the number of vehicles can be seen as a direct proxy for the vehicle density on the track. The relationship between the number of vehicles and the intensity of the waves as measured as velocity standard deviation is seen in Figure 12a. This shows a general negative correlation between the number of vehicles on the track, and the intensity of the waves observed when instruction set I is given. However, when instruction set II is given, the more aggressive driving behavior is consistent with the findings of Sugiyama, et al. 41] who report that waves are more prevalent at higher densities.

The effect of traffic waves on fuel rate is seen in Figure $12 \mathrm{~b}$, where a clear increasing trend is observed between the velocity standard deviation and the average fuel rate. This result indicates that oscillatory traffic with a high velocity standard deviation is bad from a fuel rate standpoint.

\section{Conclusion}

This article describes a set of eight experiments in which 19 to 22 vehicles drive in a ring and traffic waves emerge. Each vehicle is instrumented with an OBD-II scanner to log the fuel rate throughout each experiment, providing a link between traffic waves and fuel consumption. Trajectory data is extracted via an image processing algorithm that produces accurate trajectories useful for microscopic traffic flow modeling and aid in understanding of the onset of oscillatory traffic. The datasets and the source code used to implement the processing algorithms are also published with this work.

The results of the experiments indicate that the onset of oscillatory traffic conditions depends on driver behaviors. Specifically, experiments in which drivers were instructed to drive more aggressively produced larger traffic oscillations compared to those in which such instructions were not given. Furthermore, we demonstrate that the behavior of a single vehicle in the flow is sufficient to significantly alter the overall traffic flow. This indicates that a single vehicle, if properly driven, could potentially reduce the severity of oscillatory traffic.

\section{Acknowledgements}

This material is based upon work supported by the National Science Foundation under Grant No. CNS1446715 (B.P.), CNS-1446690 (B.S.), CNS-1446435 (J.S.), and CNS-1446702 (D.W.). The authors thank 

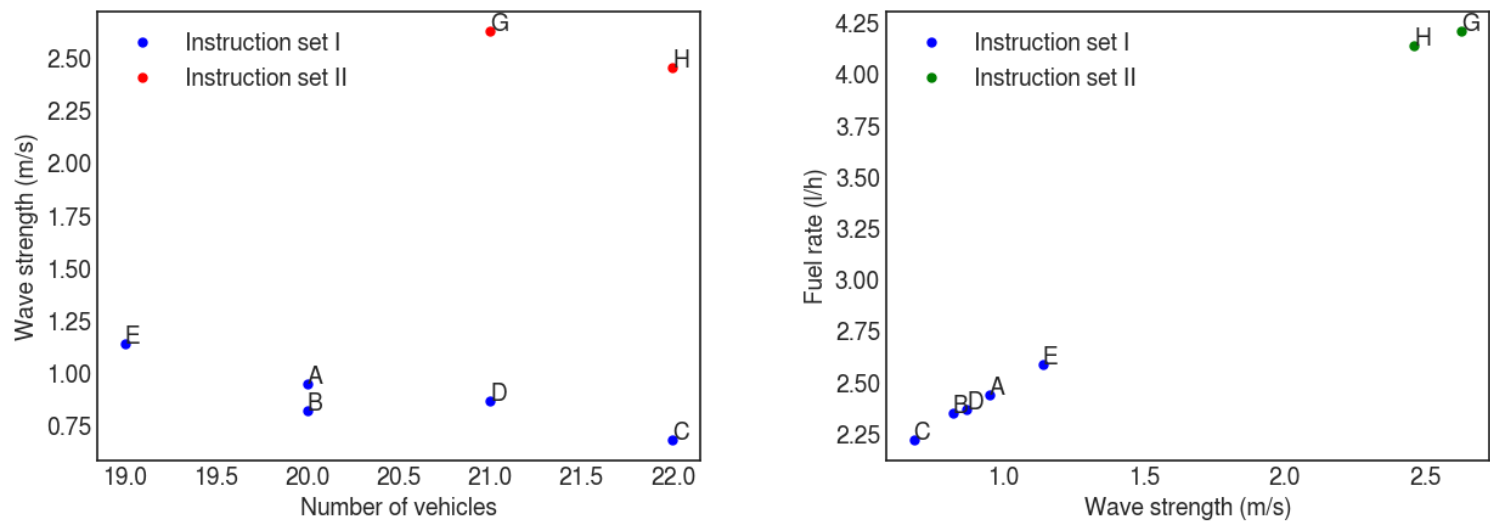

(a) Wave strength as a function of the number of vehicles (b) Fuel rate as a function of wave strength indicating on the circular track showing: $(i)$ generally decreasing that when stronger traffic waves (greater velocity stanwave strength with increasing vehicle density for instruc- dard deviation) are present, vehicles consume more fuel. tion set I, and increasing wave strength with density for instruction set II, and (ii) instruction set II generates stronger waves than instruction set I.

Figure 12: Summary of experiments depicting: $(i)$ a general decreasing trend in wave strength as number of vehicles on the track increases for instruction set I, and increasing wave strength with number of vehicles for instruction set II, (ii) instruction set II generates stronger waves (greater velocity standard deviation) than instruction set I, and (iii) an observed increase in fuel rate with wave strength.

the University of Arizona Motor Pool in providing the vehicle fleet. They offer additional special thanks for the services of N. Emptage in carrying out the experiment logistics.

\section{Appendix}

Included in the appendix are the full verbal instructions given to the drivers in experiment set I and set II. In addition, the appendix contains vehicle trajectories for all experiments presented in this article. Trajectory plots are independently colored by the corresponding spacing (to the rear bumper of the front vehicle), speed, acceleration, and fuel rate data. The appendix also includes specifications of each vehicle used in the experiments in Table A.1.

\section{Driver instructions}

Instructions for experiment set I: Drive as you would if you were in rush hour traffic. Follow the vehicle ahead without falling behind. However, drive as safely as would on the road. Do not pass the car in front of you. Do not hit the car in front of you.

Instructions for experiment set I: Drive as if you were in rush hour traffic. Follow the vehicle ahead without falling behind. Do not pass the car ahead. Do not hit the car ahead. Drive safely at all times. Do not tailgate. But put an emphasis on closing up to the vehicle ahead, if a gap starts opening up. 

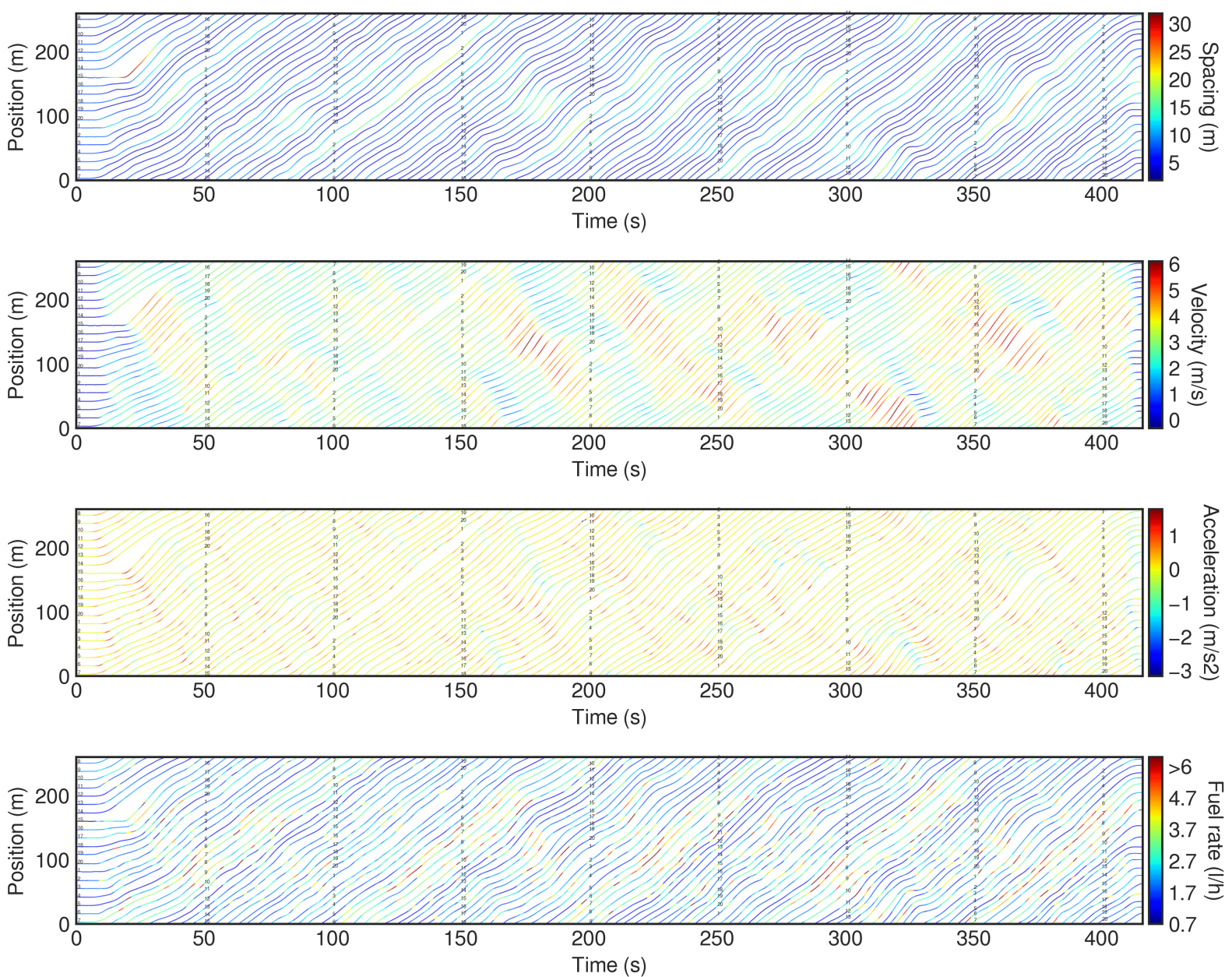

Figure A.1: Visulization of Experiment A Data. Note that the color map fuel rate plot is capped at $6 \mathrm{l} / \mathrm{h}$ for enhanced visibility. A small fraction of measurements exceed $6 \mathrm{l} / \mathrm{h}$, the maximum of which reaches $10.34 \mathrm{l} / \mathrm{h}$. Black color indicates that no data is recorded. 

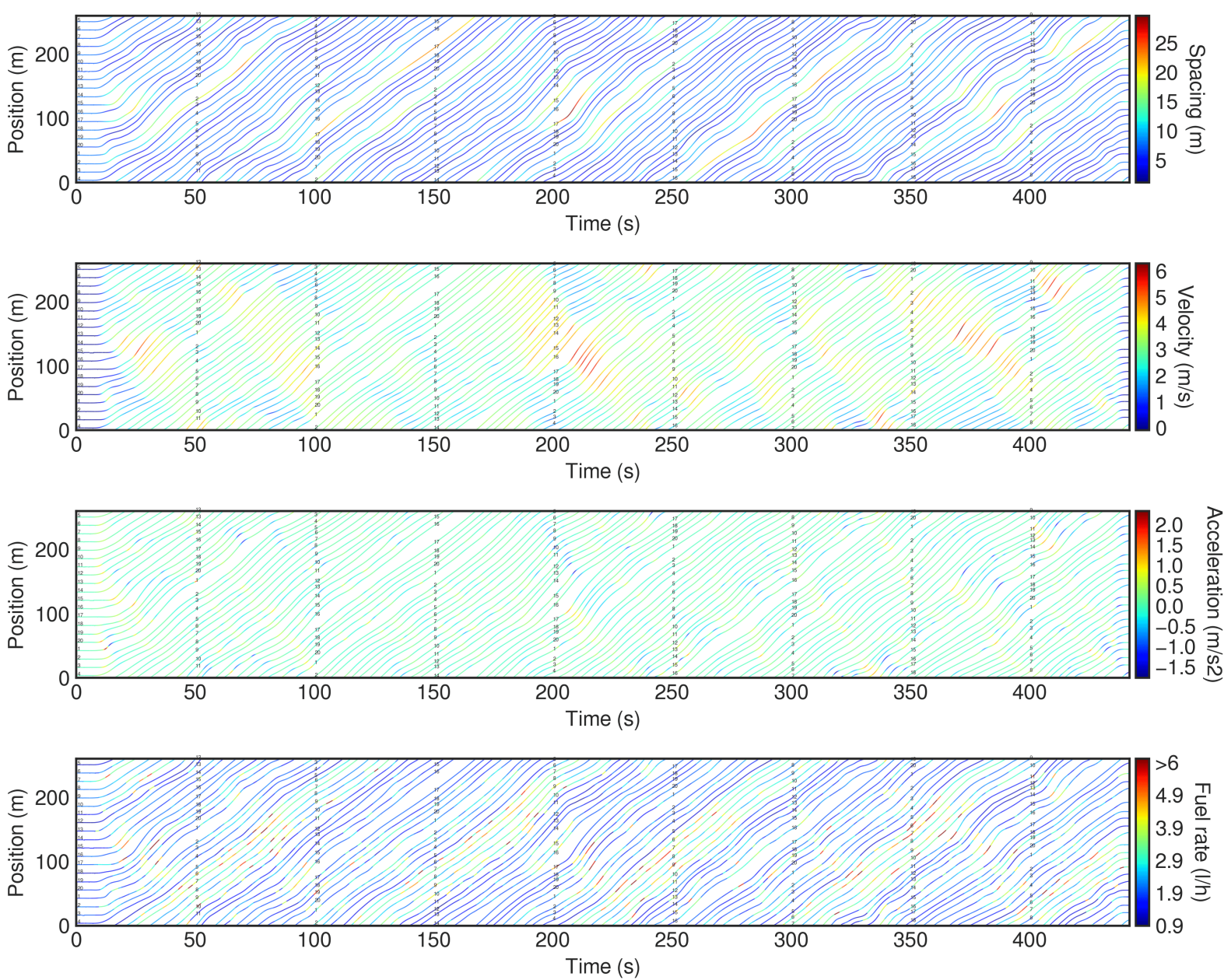

Figure A.2: Visulization of Experiment B Data. Note that the color map fuel rate plot is capped at $6 \mathrm{l} / \mathrm{h}$ for enhanced visibility. A small fraction of measurements exceed $6 \mathrm{l} / \mathrm{h}$, the maximum of which reaches $9.38 \mathrm{l} / \mathrm{h}$. Black color indicates that no data is recorded. 

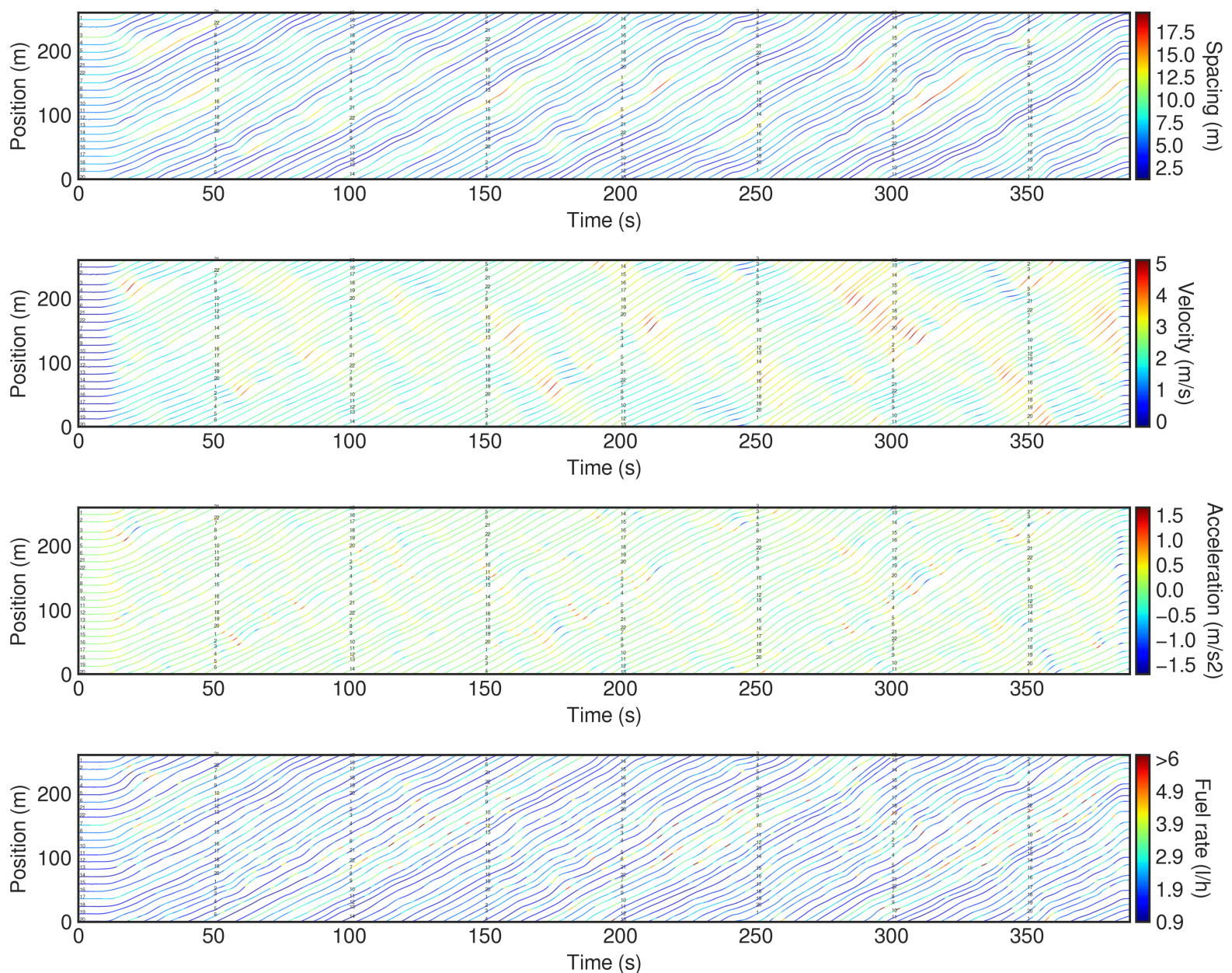

Figure A.3: Visulization of Experiment C Data. Note that the color map fuel rate plot is capped at $6 \mathrm{l} / \mathrm{h}$ for enhanced visibility. A small fraction of measurements exceed $6 \mathrm{l} / \mathrm{h}$, the maximum of which reaches $12.71 \mathrm{l} / \mathrm{h}$. Black color indicates that no data is recorded. 

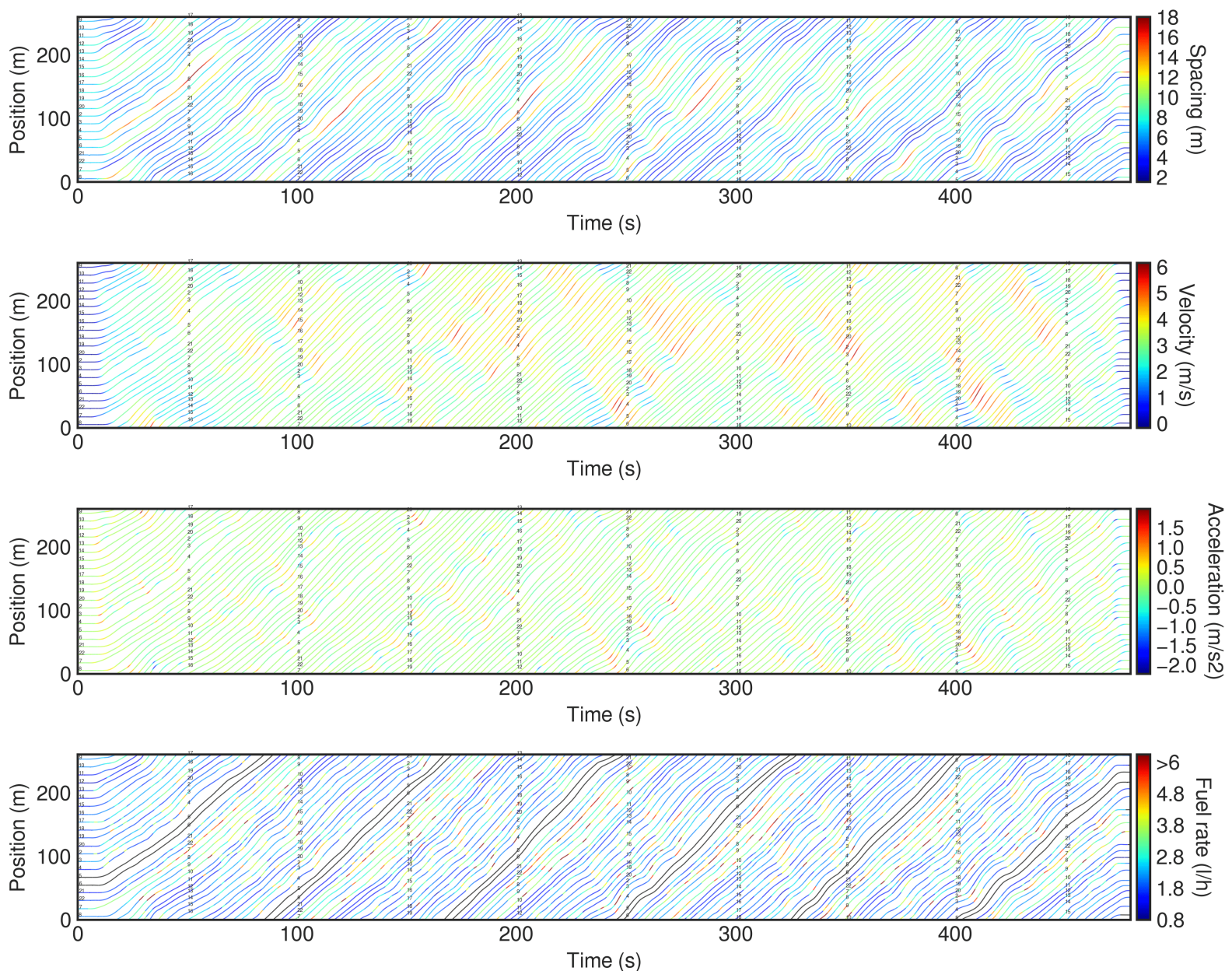

Figure A.4: Visulization of Experiment D Data. Note that the color map fuel rate plot is capped at $6 \mathrm{l} / \mathrm{h}$ for enhanced visibility. A small fraction of measurements exceed $6 \mathrm{l} / \mathrm{h}$, the maximum of which reaches $13.65 \mathrm{l} / \mathrm{h}$. Black color indicates that no data is recorded. 

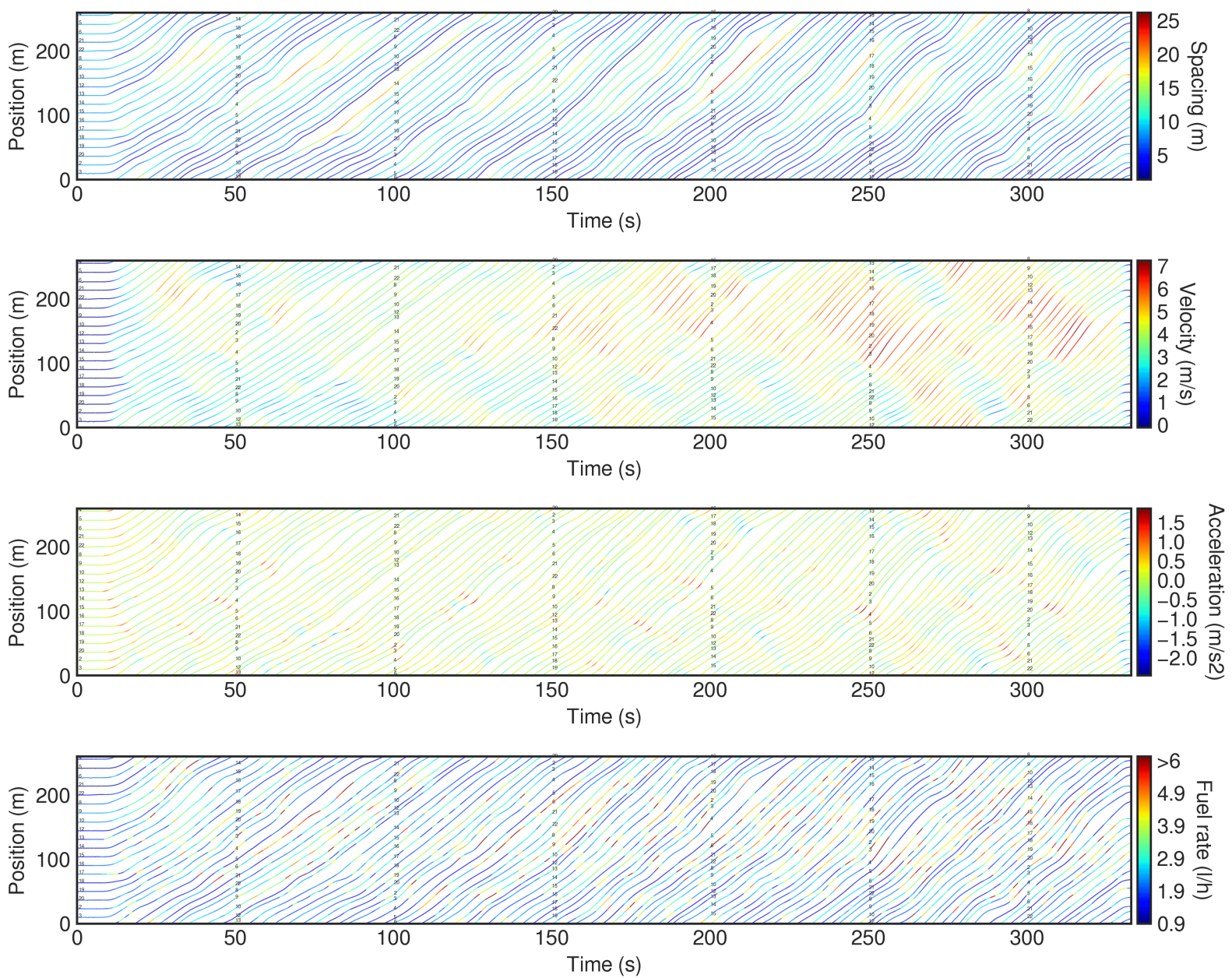

Figure A.5: Visulization of Experiment E Data. Note that the color map fuel rate plot is capped at $6 \mathrm{l} / \mathrm{h}$ for enhanced visibility. A small fraction of measurements exceed $6 \mathrm{l} / \mathrm{h}$, the maximum of which reaches $15.65 \mathrm{l} / \mathrm{h}$. Black color indicates that no data is recorded. 

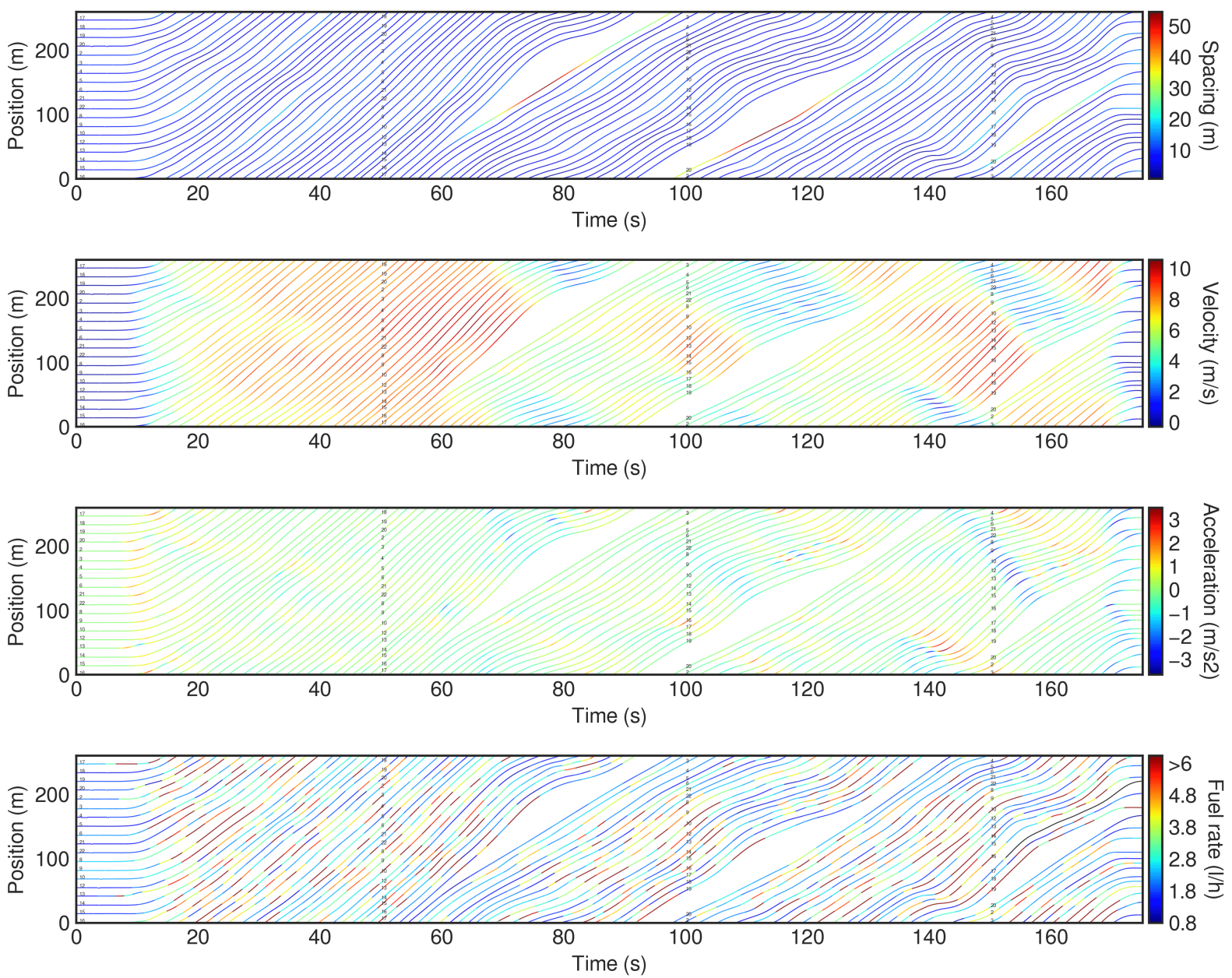

Figure A.6: Visulization of Experiment F Data. Note that the color map fuel rate plot is capped at $6 \mathrm{l} / \mathrm{h}$ for enhanced visibility. A small fraction of measurements exceed $6 \mathrm{l} / \mathrm{h}$, the maximum of which reaches $22.12 \mathrm{l} / \mathrm{h}$. Black color indicates that no data is recorded. 

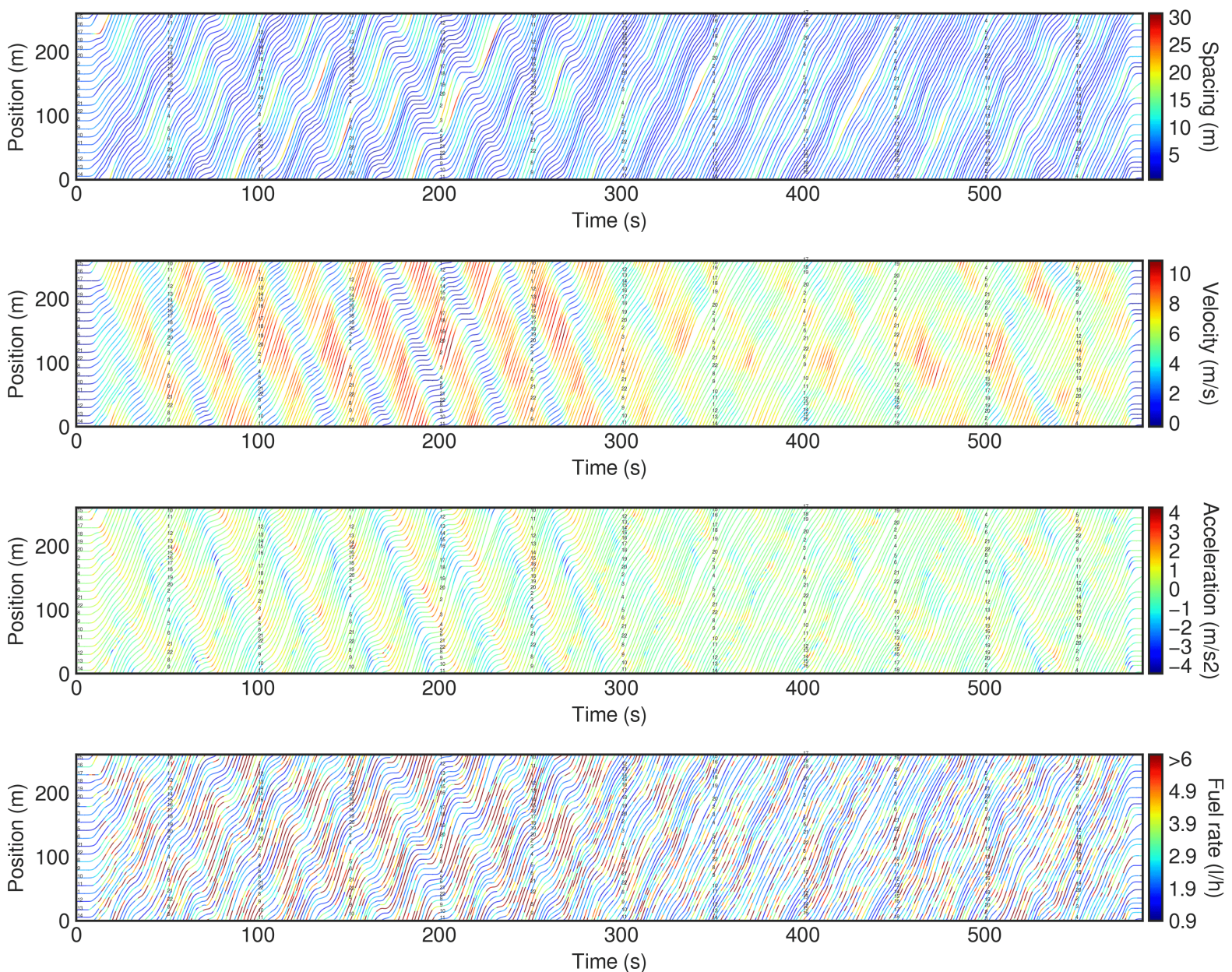

Figure A.7: Visulization of Experiment G Data. Note that the color map fuel rate plot is capped at $6 \mathrm{l} / \mathrm{h}$ for enhanced visibility. A small fraction of measurements exceed $6 \mathrm{l} / \mathrm{h}$, the maximum of which reaches $23.67 \mathrm{l} / \mathrm{h}$. Black color indicates that no data is recorded. 

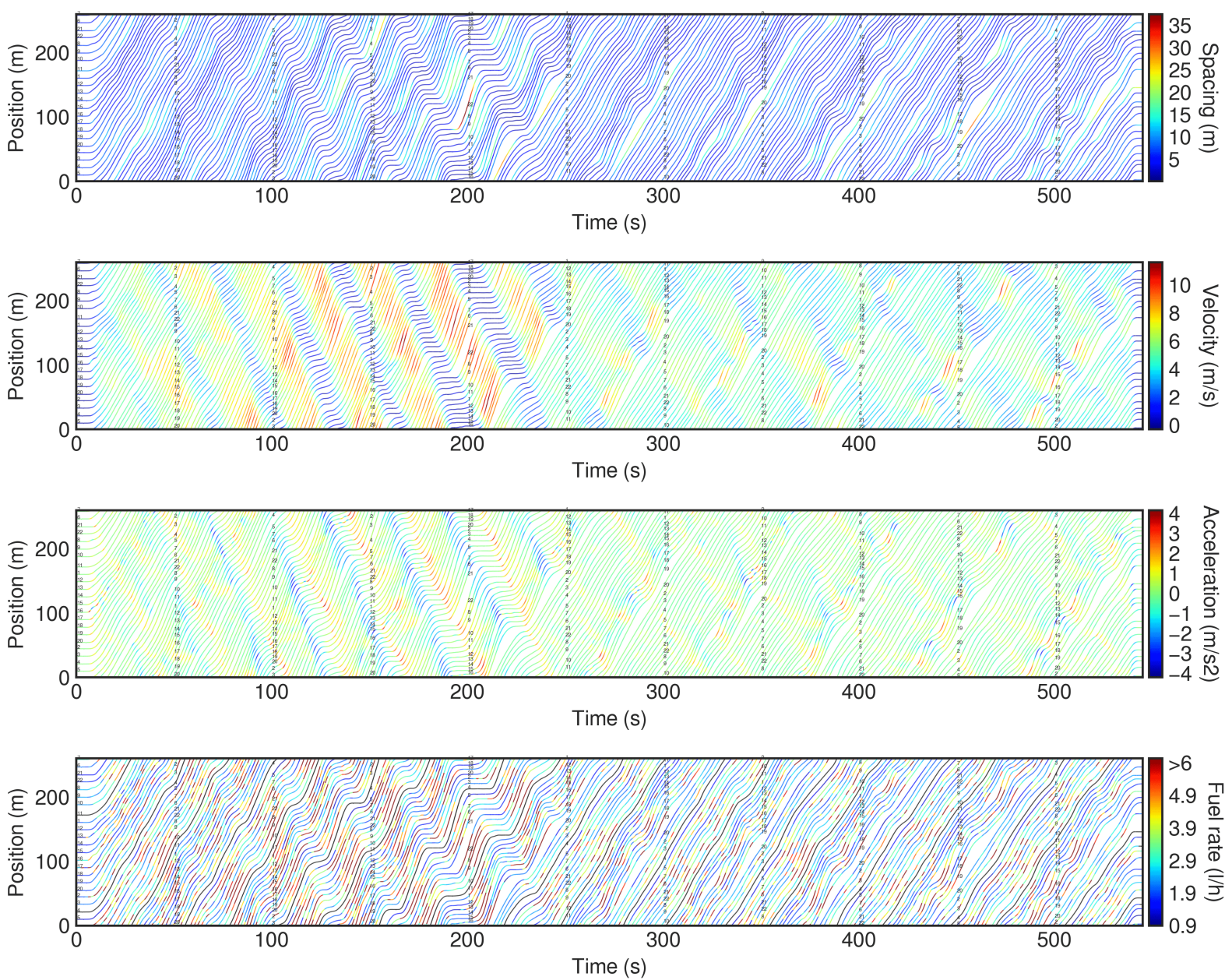

Figure A.8: Visulization of Experiment H Data. Note that the color map fuel rate plot is capped at $6 \mathrm{l} / \mathrm{h}$ for enhanced visibility. A small fraction of measurements exceed $6 \mathrm{l} / \mathrm{h}$, the maximum of which reaches $23.46 \mathrm{l} / \mathrm{h}$. Black color indicates that no data is recorded. 


\begin{tabular}{ccccccc}
\hline $\begin{array}{c}\text { Veh. } \\
\text { Num. }\end{array}$ & Year & Make & Model & $\begin{array}{c}\text { Length } \\
(\mathbf{m})\end{array}$ & $\begin{array}{c}\text { Consumption } \\
\text { City } \\
(\ell / 100 k m)\end{array}$ & $\begin{array}{c}\text { Consumption } \\
\text { Hwy. } \\
(\ell / 100 k m)\end{array}$ \\
\hline 1 & 2013 & Dodge & Grand Caravan & 5.15 & 13.83 & 9.41 \\
2 & 2013 & Dodge & Grand Caravan & 5.15 & 13.83 & 9.47 \\
3 & 2012 & Dodge & Grand Caravan & 5.15 & 13.83 & 9.47 \\
4 & 2012 & Chevrolet & Malibu & 4.87 & 10.69 & 7.13 \\
5 & 2014 & Dodge & Grand Caravan & 5.15 & 13.83 & 9.41 \\
6 & 2015 & Chevrolet & Suburban & 5.69 & 14.71 & 10.22 \\
7 & 2016 & Chevrolet & Express 2500 & 5.69 & 21.38 & 14.70 \\
8 & 2016 & Dodge & Grand Caravan & 5.15 & 13.83 & 9.41 \\
9 & 2013 & Chevrolet & Silverado & 5.22 & 15.67 & 10.68 \\
10 & 2014 & Chevrolet & Silverado & 5.21 & 13.07 & 9.80 \\
11 & 2014 & Chevrolet & Malibu & 4.86 & 9.41 & 6.53 \\
12 & 2014 & Chevrolet & Malibu & 4.86 & 9.41 & 6.53 \\
13 & 2016 & Chevrolet & Malibu Limited & 4.86 & 9.80 & 6.92 \\
14 & 2015 & Chevrolet & Malibu & 4.92 & 9.41 & 6.53 \\
15 & 2012 & Chevrolet & Malibu & 4.87 & 10.70 & 7.13 \\
16 & 2012 & Dodge & Grand Caravan & 5.15 & 13.83 & 9.41 \\
17 & 2013 & Chevrolet & Impala & 5.09 & 13.07 & 7.84 \\
18 & 2015 & Chevrolet & Malibu & 4.86 & 9.47 & 6.53 \\
19 & 2016 & Chevrolet & Suburban & 5.70 & 14.71 & 10.22 \\
20 & 2009 & Ford & Escape Hybrid & 4.44 & 6.92 & 7.84 \\
21 & 2014 & Chevrolet & Malibu & 4.86 & 9.41 & 6.53 \\
22 & 2012 & Chevrolet & Malibu & 4.87 & 10.70 & 7.13 \\
\hline
\end{tabular}

Table A.1: Vehicle properties of all vehicles used in the experiments. 
Experiment fleet

\begin{tabular}{|c|c|c|c|c|c|c|c|c|}
\hline $\begin{array}{l}\text { Veh. } \\
\text { Num. }\end{array}$ & $\begin{array}{c}\text { Exp. } \\
\text { A }\end{array}$ & $\begin{array}{c}\text { Exp. } \\
\text { B }\end{array}$ & $\begin{array}{c}\text { Exp. } \\
\text { C }\end{array}$ & $\begin{array}{c}\text { Exp. } \\
\text { D }\end{array}$ & $\begin{array}{c}\text { Exp. } \\
\text { E }\end{array}$ & $\begin{array}{c}\text { Exp. } \\
\text { F }\end{array}$ & $\begin{array}{c}\operatorname{Exp} . \\
\text { G }\end{array}$ & $\begin{array}{c}\text { Exp. } \\
\mathbf{H}\end{array}$ \\
\hline 1 & $x$ & $x$ & $x$ & & & & $x$ & $\times$ \\
\hline 2 & $\times$ & $\times$ & $\times$ & $\times$ & $\times$ & $\times$ & $\times$ & $\times$ \\
\hline 3 & $\times$ & $\times$ & $x$ & $x$ & $\times$ & $\times$ & $x$ & $\times$ \\
\hline 4 & $\times$ & $\times$ & $\times$ & $\times$ & $\times$ & $\times$ & $\times$ & $\times$ \\
\hline 5 & $x$ & $x$ & $x$ & $\times$ & $x$ & $\times$ & $\times$ & $\times$ \\
\hline 6 & $x$ & $\times$ & $x$ & $x$ & $x$ & $\times$ & $x$ & $\times$ \\
\hline 7 & $x$ & $x$ & $x$ & $x$ & & & & $\times$ \\
\hline 8 & $\times$ & $x$ & $x$ & $x$ & $x$ & $\times$ & $x$ & $\times$ \\
\hline 9 & $x$ & $\times$ & $x$ & $\times$ & $\times$ & $x$ & $\times$ & $\times$ \\
\hline 10 & $\times$ & $x$ & $x$ & $x$ & $x$ & $\times$ & $x$ & $\times$ \\
\hline 11 & $\times$ & $x$ & $x$ & $x$ & & & $x$ & $\times$ \\
\hline 12 & $x$ & $x$ & $x$ & $x$ & $x$ & $\times$ & $x$ & $\times$ \\
\hline 13 & $\times$ & $x$ & $x$ & $x$ & $x$ & $\times$ & $x$ & $\times$ \\
\hline 14 & $x$ & $x$ & $x$ & $x$ & $x$ & $\times$ & $x$ & $\times$ \\
\hline 15 & $x$ & $x$ & $\times$ & $x$ & $x$ & $\times$ & $x$ & $\times$ \\
\hline 16 & $\times$ & $\times$ & $x$ & $\times$ & $\times$ & $\times$ & $\times$ & $\times$ \\
\hline 17 & $x$ & $x$ & $x$ & $x$ & $x$ & $\times$ & $x$ & $x$ \\
\hline 18 & $\times$ & $x$ & $x$ & $x$ & $x$ & $\times$ & $x$ & $\times$ \\
\hline 19 & $\times$ & $x$ & $x$ & $x$ & $x$ & $\times$ & $x$ & $\times$ \\
\hline 20 & $x$ & $x$ & $x$ & $x$ & $x$ & $x$ & $x$ & $\times$ \\
\hline 21 & & & $x$ & $x$ & $x$ & $x$ & $x$ & $x$ \\
\hline 22 & & & $x$ & $x$ & $\times$ & $x$ & $x$ & $\times$ \\
\hline
\end{tabular}

Table A.2: Vehicles used for each experiment. An $\times$ appears of a vehicle was used for a given experiment. Each vehicle has a single unique driver over all experiments. 


\section{References}

[1] B. Greenshields. The photographic method of studying traffic behavior. In Highway Research Board Proceedings, volume 13,1934

[2] D. C. Gazis and R. S. Foote. Surveillance and control of tunnel traffic by an on-line digital computer. Transportation Science, 3(3):255-275, 1969.

[3] N. E. Nahi and A. N. Trivedi. Recursive estimation of traffic variables: Section density and average speed. Transportation Science, $7(3): 269-286,1973$.

[4] M. S. Grewal and H. J. Payne. Identification of parameters in a freeway traffic model. IEEE Transactions on systems, man, and cybernetics, SMC-6(3):176-185, 1976.

[5] Performance measurement system database. http://pems.dot.ca.gov/

[6] C. C. De Wit, F. Morbidi, L. Leon Ojeda, A. Y. Kibangou, I. Bellicot, and P. Bellemain. Grenoble traffic lab: An experimental platform for advanced traffic monitoring and forecasting [applications of control]. IEEE Control Systems, $35(3): 23-39,2015$.

[7] Minnesota traffic observatory: Data acquisition and sensing. http://www.its.umn.edu/ProgramsLabs/ MNTrafficObservatory/, 2017.

[8] A. D. May, R. Cayford, B. Coifman, and G. Merritt. Loop detector data collection and travel time measurement in the berkeley highway laboratory. California Partners for Advanced Transit and Highways (PATH), 2003.

[9] G. Dervisoglu, G. Gomes, J. Kwon, R. Horowitz, and P. Varaiya. Automatic calibration of the fundamental diagram and empirical observations on capacity. In Proceedings of the 88th Transportation Research Board Annual Meeting, volume 15, 2009.

[10] G. Dervisoglu, A. Kurzhanskiy, G. Gomes, and R. Horowitz. Macroscopic freeway model calibration with partially observed data, a case study. In Proceedings of the American Control Conference, 2014, pages 3096-3103. IEEE, 2014.

[11] J. Hueper, G. Dervisoglu, A. Muralidharan, G. Gomes, R. Horowitz, and P. Varaiya. Macroscopic modeling and simulation of freeway traffic flow. IFAC Proceedings Volumes, 42(15):112-116, 2009.

[12] A. Zeroual, N. Messai, S. Kechida, and F. Hamdi. Calibration and validation of a switched linear macroscopic traffic model. In Proceedings of the 3rd International Conference on Control, Engineering E Information Technology (CEIT), pages 1-5. IEEE, 2015.

[13] M. Bando, K. Hasebe, A. Nakayama, A. Shibata, and Y. Sugiyama. Dynamical model of traffic congestion and numerical simulation. Physical Review E, 51(2):1035, 1995.

[14] D. Helbing and B. Tilch. Generalized force model of traffic dynamics. Physical Review E, 58(1):133, 1998.

[15] Y. Hwasoo and A. Skabardonis. Understanding stop-and-go traffic in view of asymmetric traffic theory. In Transportation and Traffic Theory 2009: Golden Jubilee, pages 99-115. Springer, 2009.

[16] G. Orosz, E. Wilson, R. Szalai, and G Stépán. Exciting traffic jams: nonlinear phenomena behind traffic jam formation on highways. Physical Review E, 80(4):046205, 2009

[17] A. Tordeux and A. Seyfried. Collision-free nonuniform dynamics within continuous optimal velocity models. Physical Review E, 90(4):042812, 2014.

[18] J. Treiterer. Investigation of traffic dynamics by aerial photogrammetry techniques. final report ees278. Transportation Research Center, Department of Civil Engineering, Ohio State University, 1975.

[19] B. Coifman. Time space diagrams for thirteen shock waves. California Partners for Advanced Transit and Highways (PATH), 1997.

[20] S. A. Smith. Freeway data collection for studying vehicle interactions-technical report. final report. Technical report, JHK \& Associates, 1985.

[21] M. Schreuder, S. P. Hoogendoorn, H. J. Van Zulyen, B. Gorte, and G. Vosselman. Traffic data collection from aerial imagery. In Proceedings of the Conference on Intelligent Transportation Systems, 2003, volume 1, pages 779-784. IEEE, 2003.

[22] Federal Highway Administration. Next Generation Simulation (NGSIM). trafficanalysistools/ngsim.htm, 2017.

[23] M. Treiber, A. Kesting, and C. Thiemann. How much does traffic congestion increase fuel consumption and emissions? applying a fuel consumption model to the NGSIM trajectory data. In Proceedings of the 87th Annual Meeting of the Transportation Research Board, 2008.

[24] B. Piccoli, K. Han, T. L. Friesz, T. Yao, and J. Tang. Second-order models and traffic data from mobile sensors. Transportation Research Part C: Emerging Technologies, 52:32-56, 2015.

[25] Y. Fang, Z. Shi, and J. Cao. Calibration of an interrupted traffic flow system using ngsim trajectory data sets. In Proceedings of the 11th World Congress on Intelligent Control and Automation (WCICA), pages 4887-4892. IEEE, 2014.

[26] D. Duong, F. Saccomanno, and B. Hellinga. Calibration of microscopic traffic model for simulating safety performance. In Proceedings of the 89th Annual Meeting of the Transportation Research Board, 2010.

[27] X.-Y. Lu, P. Varaiya, and R. Horowitz. Fundamental diagram modelling and analysis based ngsim data. IFAC Proceedings Volumes, 42(15):367-374, 2009

[28] A. Kesting and M. Treiber. Calibrating car-following models by using trajectory data: Methodological study. Transportation Research Record: Journal of the Transportation Research Board, 2088:148-156, 2008.

[29] C. Thiemann, M. Treiber, and A. Kesting. Estimating acceleration and lane-changing dynamics from next generation simulation trajectory data. Transportation Research Record: Journal of the Transportation Research Board, 2088:90-101, 2008. 
[30] M. Montanino and V. Punzo. Trajectory data reconstruction and simulation-based validation against macroscopic traffic patterns. Transportation Research Part B: Methodological, 80:82-106, 2015.

[31] V. Punzo, M. T. Borzacchiello, and B. Ciuffo. On the assessment of vehicle trajectory data accuracy and application to the next generation simulation (NGSIM) program data. Transportation Research Part C: Emerging Technologies, 19(6):1243-1262, 2011.

[32] A. Kesting and M. Treiber. Calibrating car-following models by using trajectory data: Methodological study. Transportation Research Record: Journal of the Transportation Research Board, 2088:148-156, 2008.

[33] L. Vasconcelos, L. Neto, S. Santos, A. B. Silva, and Á. Seco. Calibration of the gipps car-following model using trajectory data. Transportation Research Procedia, 3:952-961, 2014.

[34] V. Punzo, D. Formisano, and V. Torrieri. Part 1: Traffic flow theory and car following: Nonstationary kalman filter for estimation of accurate and consistent car-following data. Transportation Research Record: Journal of the Transportation Research Board, 1934:1-12, 2005.

[35] Virginia Technical University Naturalistic Driving Study data. http://www.vtti.vt.edu/facilities/data-center.html

[36] R. D. Foss and A. H. Goodwin. Distracted driver behaviors and distracting conditions among adolescent drivers: findings from a naturalistic driving study. Journal of Adolescent Health, 54(5):S50-S60, 2014.

[37] G. M. Fitch, S. A. Soccolich, F. Guo, J. McClafferty, Y. Fang, R. L. Olson, M. A. Perez, R. J. Hanowski, J. M. Hankey, and T. A. Dingus. The impact of hand-held and hands-free cell phone use on driving performance and safety-critical event risk. Technical report, Virginia Polytechnic Institute and State University, 2013.

[38] J. Montgomery, K. D. Kusano, and H. C. Gabler. Age and gender differences in time to collision at braking from the 100-car naturalistic driving study. Traffic injury prevention, 15(sup1):S15-S20, 2014.

[39] University of Michigan Naturalistic Driving Data. http://www.umtri.umich.edu/our-focus/ naturalistic-driving-data.

[40] J. C. Herrera, D. B. Work, R. Herring, X. J. Ban, Q. Jacobson, and A. M. Bayen. Evaluation of traffic data obtained via GPS-enabled mobile phones: The Mobile Century field experiment. Transportation Research Part C: Emerging Technologies, 18(4):568-583, 2010.

[41] Y. Sugiyama, M. Fukui, M. Kikuchi, K. Hasebe, A. Nakayama, K. Nishinari, S. i. Tadaki, and S. Yukawa. Traffic jams without bottlenecks-experimental evidence for the physical mechanism of the formation of a jam. New Journal of Physics, 10(3):033001, 2008.

[42] S.-i. Tadaki, M. Kikuchi, M. Fukui, A. Nakayama, K. Nishinari, A. Shibata, Y. Sugiyama, T. Yosida, and S. Yukawa. Phase transition in traffic jam experiment on a circuit. New Journal of Physics, 15(10):103034, 2013.

[43] D. Shamoto, A. Tomoeda, R. Nishi, and K. Nishinari. Car-following model with relative-velocity effect and its experimental verification. Physical Review E, 83(4):046105, 2011.

[44] R. Jiang, M.-B. Hu, H. M. Zhang, Z.-Y. Gao, B. Jia, Q.-S. Wu, B. Wang, and M. Yang. Traffic experiment reveals the nature of car-following. PLOS ONE, 9(4):1-9, 042014.

[45] G. McGregor. On Microscopic Traffic Models, Intersections and Fundamental Diagrams. PhD thesis, University of Victoria, 2013.

[46] J. Tian, G. Li, M. Treiber, R. Jiang, N. Jia, and S. Ma. Cellular automaton model simulating spatiotemporal patterns, phase transitions and concave growth pattern of oscillations in traffic flow. Transportation Research Part B: Methodological, 93:560-575, 2016.

[47] L. C. Davis. Effect of adaptive cruise control systems on traffic flow. Physical Review E, 69(6):066110, 2004.

[48] A. Talebpour and H. S. Mahmassani. Influence of connected and autonomous vehicles on traffic flow stability and throughput. Transportation Research Part C: Emerging Technologies, 71:143-163, 2016.

[49] M. Guériau, R. Billot, N.-E. El Faouzi, J. Monteil, F. Armetta, and S. Hassas. How to assess the benefits of connected vehicles? a simulation framework for the design of cooperative traffic management strategies. Transportation research part C: Emerging Technologies, 67:266-279, 2016.

[50] M. Wang, W. Daamen, S. P. Hoogendoorn, and B. van Arem. Cooperative car-following control: Distributed algorithm and impact on moving jam features. IEEE Transactions on Intelligent Transportation Systems, 17(5):1459-1471, 2016.

[51] W. Levine and M. Athans. On the optimal error regulation of a string of moving vehicles. IEEE Transactions on Automatic Control, 11(3):355-361, 1966.

[52] D. Swaroop and J. K. Hedrick. String stability of interconnected systems. IEEE Transactions on Automatic Control, 41(3):349-357, 1996.

[53] S. E. Shladover. Review of the state of development of advanced vehicle control systems (avcs). Vehicle System Dynamics, 24(6-7):551-595, 1995

[54] R. E. Fenton and R. J. Mayhan. Automated highway studies at the Ohio State University-an overview. IEEE transactions on Vehicular Technology, 40(1):100-113, 1991.

[55] S. Darbha and K.R. Rajagopal. Intelligent cruise control systems and traffic flow stability. Transportation Research Part C: Emerging Technologies, 7(6):329 - 352, 1999.

[56] B. Besselink and K. H. Johansson. String stability and a delay-based spacing policy for vehicle platoons subject to disturbances. arXiv preprint arXiv:1702.01031, 2017.

[57] P. Ioannou, Z. Xu, S. Eckert, D. Clemons, and T. Sieja. Intelligent cruise control: theory and experiment. In Proceedings of the 32nd IEEE Conference on Decision and Control, pages 1885-1890. IEEE, 1993.

[58] M. Buehler, K. Iagnemma, and S. Singh. The DARPA urban challenge: autonomous vehicles in city traffic, volume 56. Springer, 2009.

[59] R. Rajamani, S. B. Choi, J. K. Law, B. K.and Hedrick, R. Prohaska, and P. Kretz. Design and experimental implementation of control for a platoon of automated vehicles. AMSE Journal of Dynamic Systems, Measurement, and Control, 
122(3):470-476, 1998.

[60] A. Bose and P. A. Ioannou. Analysis of traffic flow with mixed manual and semiautomated vehicles. IEEE Transactions on Intelligent Transportation Systems, 4(4):173-188, 2003.

61] F. Wu, R. E. Stern, S. Cui, M. L. Delle Monache, R. Bhadani, M. Bunting, M. Churchill, N. Hamilton, R. Haulcy, H. Pohlmann, B. Piccoli, B. Seibold, J. Sprinkle, and D. B. Work. Data and source code. https://github.com/Lab-Work/ TR-C2017_360Vision 2016.

[62] A. Nakayama, M. Fukui, M. Kikuchi, K. Hasebe, K. Nishinari, Y. Sugiyama, S.-i. Tadaki, and S. Yukawa. Metastability in the formation of an experimental traffic jam. New Journal of Physics, 11(8):083025, 2009.

[63] F. Wu. Panoramic Vision Source Code. https://github.com/Lab-Work/TR-C_2017_Panoramic_Vision

[64] R. E. Stern, S. Cui, M. L. Delle Monache, R. Bhadani, M. Bunting, M. Churchill, N. Hamilton, R. Haulcy, H. Pohlmann, F. Wu, B. Piccoli, B. Seibold, J. Sprinkle, and D. Work. Dissipation of stop-and-go waves via control of autonomous vehicles: Field experiments. Submited to Transportation Research part C: Emerging Technologies, 2017.

[65] D. Forsyth and J. Ponce. Computer vision: a modern approach. Pearson, 2015.

[66] S. S. Blackman and R. Popoli. Design and analysis of modern tracking systems. Artech House, 1999.

[67] Y. Bar-Shalom, T. Kirubarajan, and X.-R. Li. Estimation with applications to tracking and navigation: Wiley, 2007.

[68] N. Wang and D.-Y. Yeung. Learning a deep compact image representation for visual tracking. In C. J. C. Burges, L. Bottou, M. Welling, Z. Ghahramani, and K. Q. Weinberger, editors, Advances in Neural Information Processing Systems 26, pages 809-817. Curran Associates, Inc., 2013.

[69] H. Nam and B. Han. Learning multi-domain convolutional neural networks for visual tracking. Proceedings of the IEEE Conference on Computer Vision and Pattern Recognition (CVPR), 2016.

[70] A. Milan, S. H. Rezatofighi, A. Dick, I. Reid, and K. Schindler. Online multi-target tracking using recurrent neural networks, 2016.

[71] F. Wu, R. E. Stern, M. Churchill, M. L. Delle Monache, K. Han, B. Piccoli, and D. B. Work. Measuring trajectories and fuel consumption in oscillatory traffic: Experimental results. Proceedings of the 96th Transportation Research Board Meeting Annual Meeting, 2017.

[72] R. H. Pena-Gonzalez and M. A. Nuno-Maganda. Computer vision based real-time vehicle tracking and classification system. Proceedings of the 57th IEEE International Midwest Symposium on Circuits and Systems (MWSCAS), 2014.

[73] D. Ponsa and A. Lpez. Cascade of classifiers for vehicle detection. Lecture Notes in Computer Science (including subseries Lecture Notes in Artificial Intelligence and Lecture Notes in Bioinformatics), 4678 LNCS:980-989, 2007. cited By 11.

[74] A. J. Lipton, H. Fujiyoshi, and Raju S. Patil. Moving target classification and tracking from real-time video. In Proceedings of the Fourth IEEE Workshop on Applications of Computer Vision, pages 8-14. IEEE, 1998.

[75] C. Aydos, B. Hengst, and W. Uther. Kalman filter process models for urban vehicle tracking. In Proceedings of the 12th IEEE International Conference on Intelligent Transportation Systems, pages 1-8, Oct 2009.

[76] K. T. K. Teo, R. K. Y. Chin, N. S. V. K. Rao, F. Wong, and W. L. Khong. Vehicle tracking using particle filter for parking management system. Proceedings of the 4 th International Conference on Artificial Intelligence with Applications in Engineering and Technology, pages 193-198, Dec 2014.

[77] H. Liu, F. Sun, and K. He. Symmetry-aided particle filter for vehicle tracking. Proceedings 2007 IEEE International Conference on Robotics and Automation, pages 4633-4638, April 2007.

[78] S. Shruthi. Vehicle tracking using convolutional neural network. In Proceedings of the World Congress on Engineering, volume 2, 2011.

[79] Y. Zhou, H. Nejati, T.-T. Do, N.-M. Cheung, and L. Cheah. Image-based vehicle analysis using deep neural network: A systematic study. In Proceedings of the IEEE International Conference on Digital Signal Processing, 2016.

[80] V. Kastrinaki, M. Zervakis, and K. Kalaitzakis. A survey of video processing techniques for traffic applications. Image and vision computing, 21(4):359-381, 2003.

[81] N. Buch, S. A. Velastin, and J. Orwell. A review of computer vision techniques for the analysis of urban traffic. IEEE Transactions on Intelligent Transportation Systems, 12(3):920-939, 2011.

[82] J. Badenas and F. Pla. Applying computer vision techniques to traffic monitoring tasks. Lecture Notes in Computer Science Methodology and Tools in Knowledge-Based Systems, pages 776-785, 1998.

[83] G. Farneback. Two-frame motion estimation based on polynomial expansion. Lecture Notes in Computer Science, 2749:363-370, 2003.

[84] J. P. Serra. Image analysis and mathematical morphology. Academic Press, 1982.

[85] M. Ester, H.-P. Kriegel, J. Sander, and X. Xu. A density-based algorithm for discovering clusters in large spatial databases with noise. In Proceedings of the Second International Conference on Knowledge Discovery and Data Mining, pages 226231. AAAI Press, 1996.

[86] H. Edelsbrunner and R. Seidel. Voronoi diagrams and arrangements. Discrete 85 Computational Geometry, 1(1):2544, 1986.

[87] OpenCV.org. Open source computer vision library. http://opencv.org/ 2016

[88] Arie Nakhmani and Allen Tannenbaum. A new distance measure based on generalized image normalized cross-correlation for robust video tracking and image recognition. Pattern recognition letters, 34(3):315-321, 2013

[89] P. Dierckx. Algorithms for smoothing data with periodic and parametric splines. Computer Graphics and Image Processing, 20(2):171184, 1982.

[90] L. L. Schumaker and P. Dierckx. Curve and surface fitting with splines. Mathematics of Computation, 63(207):427, 1994.

[91] B. C. Russell, A. Torralba, K. P. Murphy, and W. T. Freeman. Labelme: A database and web-based tool for image annotation. International Journal of Computer Vision, 77(1-3):157-173, 2007.

[92] A. Mehar, S. Chandra, and S. Velmurugan. Speed and acceleration characteristics of different types of vehicles on multi-lane 
highways. European Transport, 55:1825-3997, 2013.

[93] U.S. Energy Information Administration. Annual energy review, 2012. 